\title{
Density fluctuations associated with the sawtooth internal disruption
}

\author{
J. Andreoletti ( $\left.{ }^{(}\right)$, C. Laviron ( $\left.{ }^{1}\right)$, J. Olivain $\left({ }^{1}\right)$, A. L. Pecquet ( ${ }^{1}$ ), F. Gervais ( ${ }^{2}$ ), \\ D. Gresillon $\left({ }^{2}\right)$, P. Hennequin $\left({ }^{2}\right)$, A. Quemeneur $\left({ }^{2}\right)$ and A. Truc $\left({ }^{2}\right)$ \\ (1) Association EURATOM-CEA sur la Fusion, DRFC-CEN-Cadarache, 13108 Saint Paul Lez \\ Durance, France \\ (2) PMI Ecole Polytechnique, 91128 Palaiseau Cedex, France
}

(Received 4 March 1991, revised 24 May 1991, accepted 30 May 1991)

\begin{abstract}
Résumé. - Des fluctuations, spécifiquement reliées à la relaxation interne en dent de scie, ont été observées sur le tokamak TFR en analysant la densité fluctuante par diffusion de la lumière émise par un laser $\mathrm{CO}_{2}$. La localisation temporelle observée est clairement liée aux phases successives du processus de relaxation. Ces fluctuations sont observables pendant le mouvement hélicoïdal « kink », mais la bouffee principale correspond à la phase d'effondrement de la température. Notre étude concerne cette forte bouffée de densité fluctuante; nous montrons d'abord les propriétés spectrales en fréquence et en nombre d'onde et la pseudo-relation de dispersion correspondante. Les fluctuations sont localisées dans l'espace; elles se situent à l'intérieur de la surface $q=1$ et leur extension azimutale est d'environ $120^{\circ}$. Compte tenu du vrillage du plasma central durant la phase de mouvement «kink» turbulent, cette localisation correspond à la position azimutale où le flux de chaleur, sortant de la zone centrale du plasma, apparaît le plus tôt et évolue le plus rapidement. Le niveau de puissance de ces fluctuations est deux ordres de grandeur plus élevé que celuı de la turbulence quasi-stationnaire locale. Ces observations sont en bon accord avec les prédictions du modèle de disruption en dent de scie proposé par Andréoletti. Les fluctuations spécifiques observées présentent plusieurs similitudes avec la turbulence « magnétodrift» décrite dans ce modèle.
\end{abstract}

\footnotetext{
Abstract. - Fluctuations specially related to the sawtooth internal disruption has been observed on TFR tokamak plasmas by analyzing the fluctuations density with $\mathrm{CO}_{2}$ laser light scattering. The time localization is clearly connected with the successive phases of the relaxation process. Some specific fluctuations appear in relation to the kink motion, but te main burst corresponds to the collapse phase. We concentrate our study on this strong burst and show first its frequency and wave number spectral properties and the corresponding pseudo dispersion relation. The fluctuations are spatially localized. They are within the interior of the $q=1$ surface and extend approximately $120^{\circ}$ azimuthally. Taking into account the twisting of the central plasma during the turbulent kink phase, this location agrees with the azimuthal position of the "sooner and faster" outgoing heat flux. The power level of these fluctuations is two orders of magnitude larger than the local quasi-stationary turbulence. These observations are in fair agreement with the
} 
predictions of the sawtooth disruption model previously proposed by Andreoletti. The observed specific fluctuations show several similarities with the so-called «magnetodrift turbulence» described in the model.

\section{Introduction.}

Since its first experimental observation by Von Goeler et al. [1], sawtooth phenomenon, also known as internal disruption, has remained an active research area. Although a lot of theoretical works has been devoted to the subject over the last fifteen years [2-31], we have to conclude that none of the theoretical models put forward so far are in agreement with all the experimental data [32]. The stability of the most probable driving mechanisms, i.e. ideal and resistive mhd kink motions, and the resistive tearing mode, have been the object of many calculations [2-12] and are reasonably well understood. The central current density profile plays an important rôle in these mechanisms, and measurements of the $q(r)$ profile result in two categories : either very flat $(q \approx 1)$ inside $r_{1}[35,36]$, or rather deep $\left(q_{0} \approx 0.8\right.$ to 0.6 for the center) [37, 38, 39] with seemingly a plateau near and inside $r_{1}[37,40,41]$ ( $r_{1}$ is the $q=1$ surface radius). Furthermore in the case of a "monster " sawtooth (regeneration period of more than $2 \mathrm{~s}$ ) there is no doubt that the current density reaches a smooth peaked profile with $q_{0}$ values much less than unity and without any plateau. Nevertheless the crash following such a long regeneration period is similar to a common one. It is even faster $[45,46]$ probably due to the higher value of the pressure gradient.

The ideal mhd internal kink in cylindrical geometry is mainly a current driven mode, unstable when $q<1$, even for a zero plasma pressure [2]. In toroidal geometry, the cylindrical terms identically cancel out, and the toroidal kink is a pressure driven instability depending on a shear integral $S$, a pressure gradient integral $\beta_{\theta}$, and coupling to a $\xi_{2}(m / n=2 / 1)$ harmonic $[3,4]$. When the safety factor $q$ has a monotonic non flat profile the calculated critical $\beta_{\theta}$ value appears to be larger than the experimental $\beta_{\theta}$ value and so the internal kink is stable. Moreover transition of an mhd system from stability to instability takes place through $\omega=0$, and so catastrophic increase of the kink motion can hardly be explained. Yet if the instability could take place, it would be limited to a small amplitude by flux conservation [18]. Even the possible occurrence of small scale ideal mhd turbulence cannot provide a solution. Due to the flux conservation and low $\beta$ energetics, the displacement and twisting of magnetic lines must remain small because of the finite magnetic shear. In the low $\beta$ dynamics, perturbations are almost divergence free and the pressure is conserved along with the motion $\mathrm{d} p / \mathrm{d} t \approx 0$. So in the frame of flux preserving low $\beta$ ideal mhd, the collapse of a finite magnetic shear configuration is impossible.

Resistivity can break the flux conservation constraint of ideal mhd, and so allows a fuller development of the nonlinear evolution explaining the crash by the squashing of the hot core itself [15]. But in a hot plasma, resistivity is weak and so evolution is slow. Furthermore, since full reconnection implies that $q_{0}$ oscillates around one, the model cannot explain sawteeth in plasmas with a central $q_{0}$ value weakly varying and much less than unity $[37,38,39]$.

Turbulence is expected to accelerate transport and also magnetic reconnection. In an early proposal by Samain [29], and Dubois et al. [30,31] the final collapse is attributed to the onset of fine scale resistive mhd turbulence, initiated by a current sheet appearing all along the two branches of the island separatrix, and propagating radially inwards and outwards.

The model proposed recently by Andreoletti [33, 34] relies on so-called « magnetodrift turbulence " initiated during the kink phase by the shear of the return velocity flux, and 
during the collapse by the pressure gradient. The model includes explanations for many aspects of the sawtooth phenomenon: the low value of the critical $\beta_{\theta}$, the catastrophic growth of the kink motion, cold bubble formation in the wake, crescent distortion of the hot core, asymmetry of the outgoing heat flux, plateau formation in the $q(r)$ profile. Plausible explanations can also be given for the large variety of the sawtooth phenomena, concerning precursor and successor oscillations, and partial sawteeth. Spectral properties, level estimates and geometrical localization of the fluctuations are obtained from the model.

Recent observations by a tomographic treatment of two orthogonal soft X-ray cameras lead to a two dimensional image of the dynamical process taking place during the relaxation [4247]. They clearly show the large scale smooth convective cells of the kink like motion. In addition to these phenomena, we have recently reported the observation of fluctuations specifically associated with the sawtooth crash [48-51]. In these reports the time synchronism and the frequency spectrum were given. In the present article a more detailed study is reported. We show that the strongest fluctuations are radially and azimuthally localized. The extension of the "fluctuations cell» and its relative localization with respect to the kink motion are given. The level is estimated relative to the quasi-stationary turbulence. As we shall explain in the discussion, the observed sawtooth specific fluctuations do not correspond to fully developed turbulence but rather to nascent turbulence structured by the production process.

\section{Experimental procedure.}

The reported experiments have been performed on the TFR tokamak, a medium size machine $(R=1 \mathrm{~m} ; a=0.2 \mathrm{~m}$ ) with a circular cross section. They concern standard ohmically heated discharges e.g. $B=4.5 \mathrm{~T}, I=240 \mathrm{kA}$, central density $9 \times 10^{19} \mathrm{~m}^{-3}$, central electron temperature of 1.2 to $1.4 \mathrm{keV}$, central ion deuterium temperature of about $0.8 \mathrm{keV}$ and edge safety factor $q_{\mathrm{a}}=3.4$. The effective plasma radius is around $18 \mathrm{~cm}$ and the estimated $q=1$ surface radius is $r_{1}=5 \mathrm{~cm}$. The $Z_{\text {eff }}$ values are typically around 2 . The discharge lasts about $0.5 \mathrm{~s}$ and the electron energy confinement time is $25 \mathrm{~ms}$.

Sawtooth behaviour is observed throughout the discharge for most operating conditions after the current profile has peaked. The main diagnostic used to study sawteeth is an array of 26 soft X-ray (SX) detectors, viewing the plasma from above. The array has a spatial resolution of about $1.5 \mathrm{~cm}$ at the midplane and views the plasma from $15 \mathrm{~cm}$ inside the equilibrium major radius to $16 \mathrm{~cm}$ outside. A brief description of this $\mathrm{SX}$ system can be found on reference [31].

Detection of the density fluctuations is made with a conventional coherent scattering apparatus usually employed to measure the quasi-stationary low frequency turbulence [52]. A $3 \mathrm{~W} \mathrm{CO} 2$ single mode laser $\left(\lambda_{\mathrm{i}}=10.6 \mu \mathrm{m}\right)$, with a beam radius $w_{0}=1.3 \mathrm{~cm}$ in the plasma is used. The beam crosses the plasma vertically and can be scanned in the major radial direction from $X=-4 \mathrm{~cm}$ to $X=+8 \mathrm{~cm}$, with the negative values corresponding to the high field side of the plasma. The collected signal is emitted from a vertical chord of the plasma. The scattering angles are variable between 0.5 to $3 \mathrm{mrad}$, thus allowing measurements of horizontal wave numbers $k$ between 3 and $20 \mathrm{~cm}^{-1}$ The detector current $i(t)$ is proportional to the instantaneous space Fourier transform, with a $\mathbf{k}$ density fluctuations wave vector. It is fed to a fast digitizer ( $100 \mathrm{MHz} ; n \times 32 \mathrm{Kwords}$ buffer) and sent to the TFR main computer. After signal processing, we get the scattered power quantitatively proportional to the Fourier transform $S_{k}(\omega)$ of the space and time autocorrelation function of the fluctuating density $\tilde{n}(r, t)$.

The scattering set up and soft X-ray port are separated toroidally by $90^{\circ}$ and comparison of 
the two measurements requires a poloidal rotation which is positive or negative, depending on the directions of the current and main magnetic field.

\section{Quasi-stationary turbulence.}

Because there is a partial overlapping between the new « specific » fluctuations and the quasistationary (QS) ones, we have to recall in some detail the main properties of the usual QS turbulence : $\omega$ and $k$ spectra, "intermittency », radial profile. The power spectrum of usual density fluctuations emitted from a plasma diameter is well approximated by four linear sections [52], separated by three frequencies $f_{1}, f_{2}, f_{3}$, in a double log scale representation: $\log S_{k}=F(\log f)$ as shown in figure 1. Although the spatial localization cannot be measured along the direction of the laser beam (vertical direction), we can make a conjecture that the spectrum is made of different portions, each associated with one particular zone. The partial spectra of these emissive zones are schematically shown in figure 1 as dashed lines. The low frequencies $\left(f \approx f_{1}\right)$ are associated with the outer zone, the intermediate frequencies $\left(f \approx f_{2}\right)$ with the gradient zone while the high frequency tail $\left(f>f_{3}\right)$ is attributed to the central region. Several subsidary observations sustain such a conjecture :

i) During some ICRF auxiliary heating experiments, a large increase of the low frequency part of the spectrum was observed every time a strong interaction of the plasma with the limiter was noticed [53], suggesting a link between the low frequency behaviour and the outer region of the plasma.

ii) When the scattering volume of observation is moved to a vertical chord away from the center of the plasma $(r / a \approx 2 / 3)$ the high frequency part of the spectrum $\left(f>f_{3}\right)$ disappears.

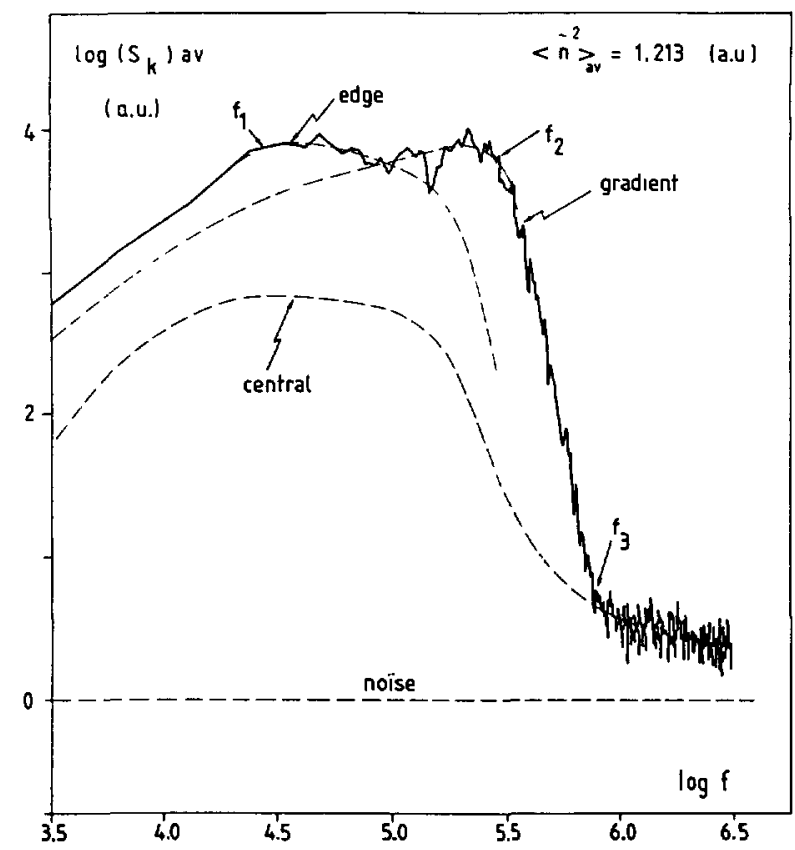

Fig. 1. - Average density fluctuations spectrum of the quasi-stationary turbulence versus frequency for a central chord $\left(k=5 \mathrm{~cm}^{-1}\right.$, frequency bandwith $\left.\Delta f=6 \mathrm{kHz}\right)$. The dotted lines correspond to conjectured spectrum associated with the different plasma zones. $\left\langle\tilde{n}^{2}\right\rangle_{\mathrm{av}}=\int\left(S_{k}\right)_{\mathrm{av}} \mathrm{d} f=$ mean quadratic value of the stationary density fluctuations. 
iii) During pellet injection experiments, ablated in the gradient zone, the fluctuations increase mainly in the intermediate frequency zone.

iv) Using phase contrast as an imaging technique for plasma density fluctuations, Weisen [54] clearly shows that the low frequency part of the spectrum preferentially stems from the outer plasma zones.

Associated $k$ spectrum variations, after frequency integration, have been previously published [52]. These exhibit an amplitude maximum for $k=5 \mathrm{~cm}^{-1}$, and two apparent decaying slopes for higher $k$ values (Fig. 2). The decaying $k^{-2.2}$ slope is considered to be significant, however the apparent second observed slope $k^{-4.5}$ is mainly due to a reduction of the scattering volume. Indeed if the fluctuation wavevectors are perpendicular to the $\mathbf{B}$ field lines, only those parts of the scattering volume where the observed $\mathbf{k} \pm \Delta \mathbf{k}$ is perpendicular to B can contribute to the signal. For $k \geqslant 20 \mathrm{~cm}^{-1}$ the relative wave number resolution $\Delta k / k \leqslant 0.07$ becomes smaller than the magnetic line angle $\alpha=B_{\mathrm{p}} / B_{\mathrm{t}}$. Then the effective scattering volume is reduced and does not coincide anymore with a full vertical chord. This effect tends to increase the $k$ exponent. The true value is somewhat smaller.

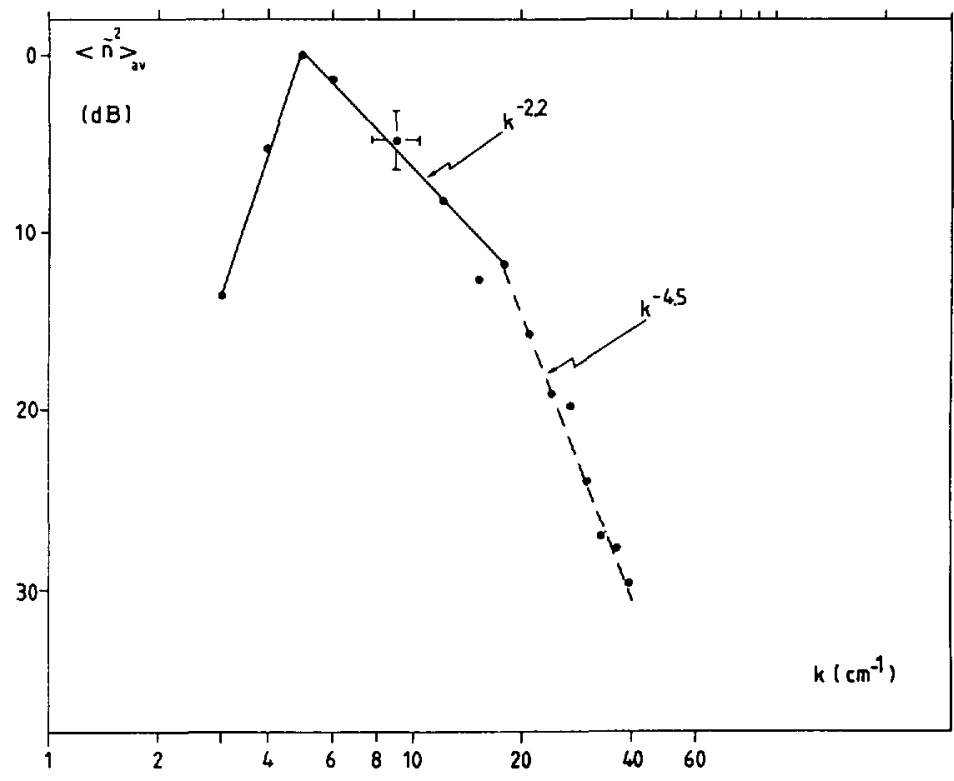

Fig. 2. - Wave number spectrum of the quasi-stationary turbulence for a central chord.

Quasi-stationary behavior is only an average property. If we examine the time sequence in more details by fragmenting the frequency spectrum, we observe "intermittency " [55]. This is not very pronounced near the intermediate frequency $f_{2}$ but it is more important in the low frequency part of the spectrum (near $f_{1}$ ). In typical TFR plasmas, the successive bursts last for $\Delta t=30$ to $60 \mu \mathrm{s}$ at intervals of time separated by 150 to $250 \mu$ s (Fig. 3).

Moreover, the fluctuations amplitude is strongly nouniform. Many experiments have shown that the relative density fluctuations are an increasing function of the radius with central values in the range $\tilde{n} / n=1$ to $3 \times 10^{-3}$ and edge values $\tilde{n} / n \geq 10^{-1}$ (see for example [56]). 


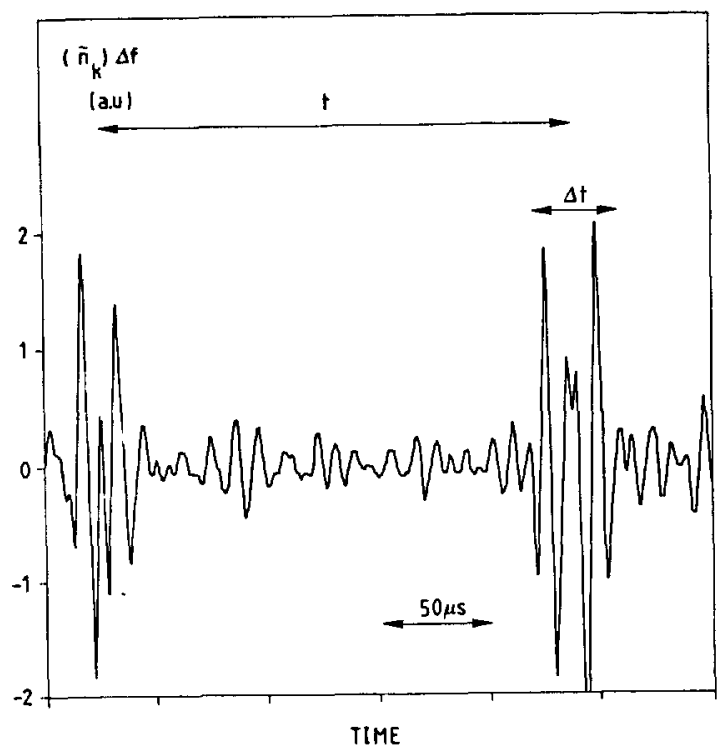

Fig. 3. - Time evolution of a filtered frequency band (Central frequency $=100 \mathrm{kHz}, \Delta f= \pm 30 \mathrm{kHz}$; $k=7 \mathrm{~cm}^{-1}$ ) of the quasi-stationary turbulence : « intermittency ».

\section{Specific density fluctuations.}

4.1 TIME ANALYSIS : THE FOUR PHASES. - During internal disruptions, « specific» density fluctuations have been detected [48]. They consist principally of a high frequency spectrum $\left(0.5 \mathrm{Mhz} \leqslant f \leqslant 2.2 \mathrm{Mhz}\right.$, for $k=7 \mathrm{~cm}^{-1}$ ) which is superimposed on the quasi-stationary turbulence previously reported. However, a more comprehensive study shows an evolution of the fluctuations during the various phases of the internal disruption as observed from the soft $\mathrm{X}$-ray radiation signals.

Four phases can be identified. The first shows a precursor oscillation and can possibly be strongly shortened. The second corresponds to a helical displacement of the hot core. The third shows a fast drop of the central emissivity with a poloidal expansion of the hot core and, in the fourth one, the plasma tends to recover its poloidal symmetry. The traces (a) to (e) in figure 4 refer to the soft X-ray signals stemming from various chords while (f) refers to the mean square amplitude of the scattering signal filtered between 0.4 to $3.1 \mathrm{Mhz}$, for $k=5 \mathrm{~cm}^{-1}$ The envelope of the high frequency signal itself is shown in figure 5 .

- An $m=1$ precursor oscillation seen on the chords (b) and (d) constitutes the first phase. No correlated particular modification appears on the density fluctuations.

- The fast displacement of the hot core is observed as a decay of the X-ray signal (b) and an increase of signal (d) indicating that the direction of the displacement is from (a) to (e). During this second phase, a noticeable increase of the scattering signal is detected. The duration of this period ranges from 15 to $20 \mu \mathrm{s}$.

- The third phase, lasting $20 \mu \mathrm{s}$, corresponds to a rapid drop of the central emissivity and to a heat pulse expelled outside the inversion radius (traces (a) and (e)). We notice an asymmetry in the process : the energy flow appears sooner and faster on chord (a) than on chord (e). During this phase, the power level of the "specific" fluctuations increases considerably. It is maximum in the middle of this period and then decreases to a value close to the one at the end of the second phase. 


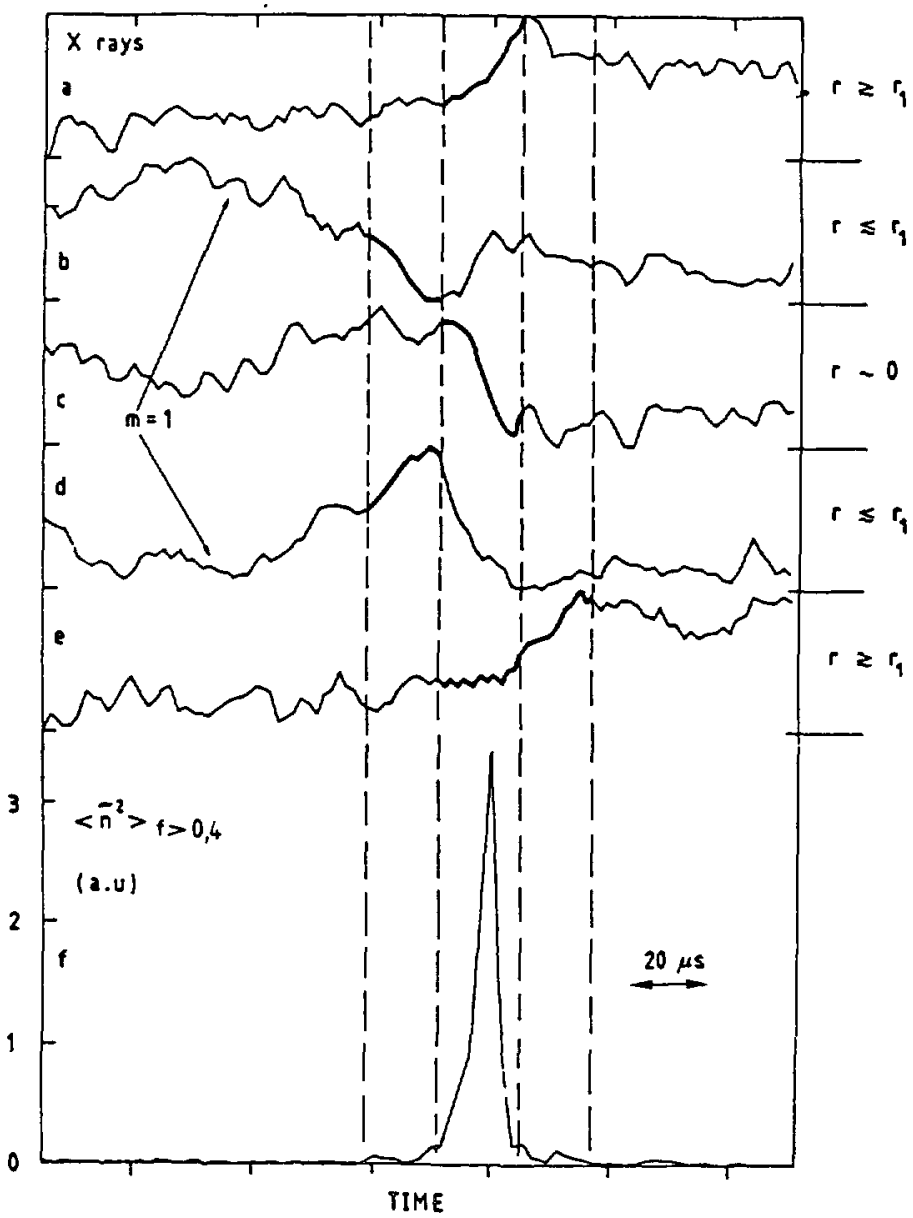

Fig. 4. - Temporal evolution of soft X-ray signals and of specific density fluctuations during the different phases of the sawtooth crash. a) to e) are soft X-ray signals for different chords from inner to outer side of the torus. $r_{1}$ is the inversion surface radius. The portions of the soft $\mathrm{X}$-ray signals characteristic of the three last phases are in bold type. $f$ ) shows the mean quadratic value of the density fluctuations filtered above $0.4 \mathrm{MHz}$ for a central chord $\left(k=5 \mathrm{~cm}^{-1}\right)$.

- The last phase is associated with a profile reorganisation. The heat flow continues on chord (e). During this last phase, covering about $20 \mu \mathrm{s}$, the level of the fluctuations is comparable to the one during the second phase. Afterwards a few small bursts can be observed, one of which is shown in figure 5.

4.2 FREQUENCY SPECTRUM IN THE FOUR PHASES. - The spectral composition of the fluctuations is not the same during the successive phases.

The weak "specific" fluctuations, clearly observed in relation to the fast helical displacement (phase 2), correspond to frequencies $f \approx f_{2}$, i.e. : $400 \mathrm{kHz} \leqslant f \leqslant 600 \mathrm{kHz}$, for $k=5 \mathrm{~cm}^{-1}$. Below $400 \mathrm{kHz}$ the observation is masked by the part of the QS spectrum attributed to the gradient zone turbulence. Nevertheless, a few examples show that an increase of the fluctuations during the same period of time can be seen down to 200 or 


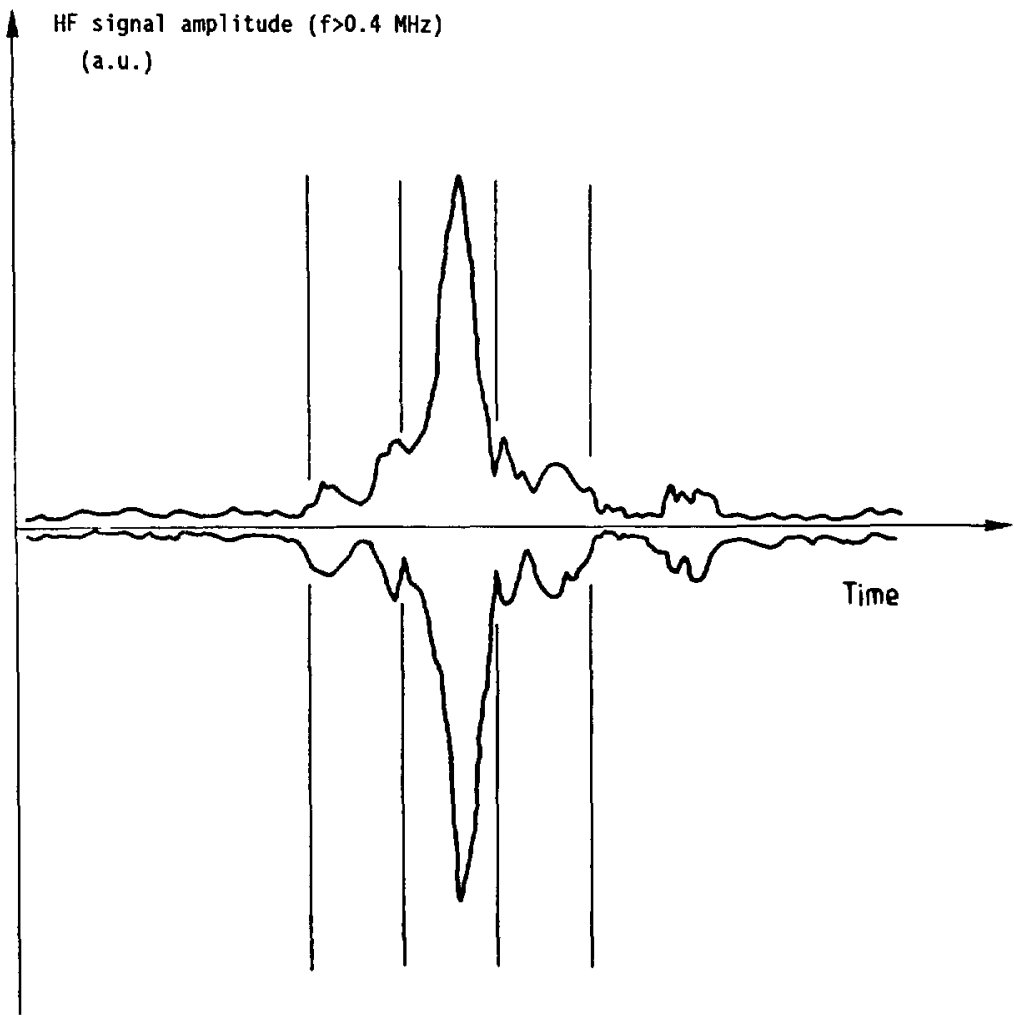

Fig. 5. - Envelope of the high frequency filtered signal $\left(f>0.4 \mathrm{MHz} ; k=5 \mathrm{~cm}^{-1}\right)$. The four datum lines correspond to those in figure 4.

$250 \mathrm{kHz}$. These observations suggest a frequency domain approximately two times lower than the one of the main burst during the collapse (see next section).

Estimation of the power level of phase 2 may also be difficult. For the part above $400 \mathrm{kHz}$, we notice : $\left\langle\tilde{n}^{2}\right\rangle_{\text {kınk }} \approx 1 / 30\left\langle\tilde{n}^{2}\right\rangle_{\text {collapse }}$ Amplitude and frequency spectrum of the fluctuations observed during the last phase (the return) are similar.

The strong burst associated with the collapse (phase 3 ) is due to frequencies greater than $f_{2}$. It will be the object of a detailed analysis presented in the next chapter.

\section{Specific fluctuations during the collapse.}

5.1 SPECTRAL PROPERTIES. - The properties of these fluctuations will be described according to two complementary approaches. Spectral analysis is used for the smaller scale properties (which are not directly accessible in space-time, and are more irregular) whereas spatio-temporal analysis will be used for the domain where the process is localized.

5.1.1 Frequency spectrum. - A $20 \mu$ s period for the scattered signal is necessary to compute a Fourier analysis and define a " quasi instantaneous » spectrum of turbulence. Excluding the time duration of the internal disruption, thirty successive "quasi instantaneous $"$ spectra preceding the crash and thirty others following it are performed, and averaged to define a mean spectrum $\left(S_{k}\right)_{\mathrm{av}}$ of the quasi-stationary turbulence (Fig. 6a). It is important to note that 


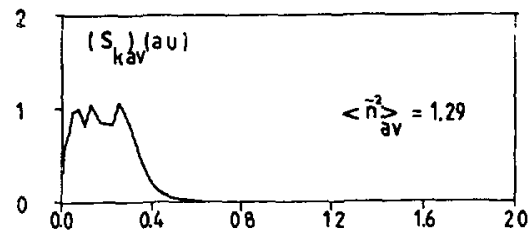

(a)

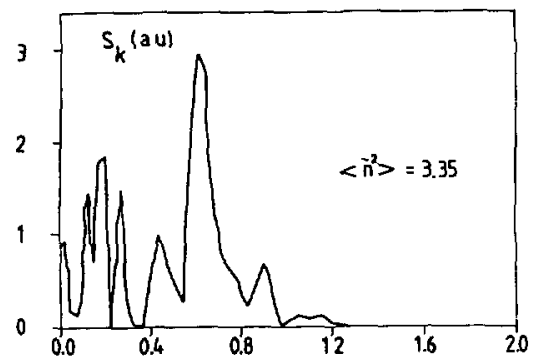

(b)

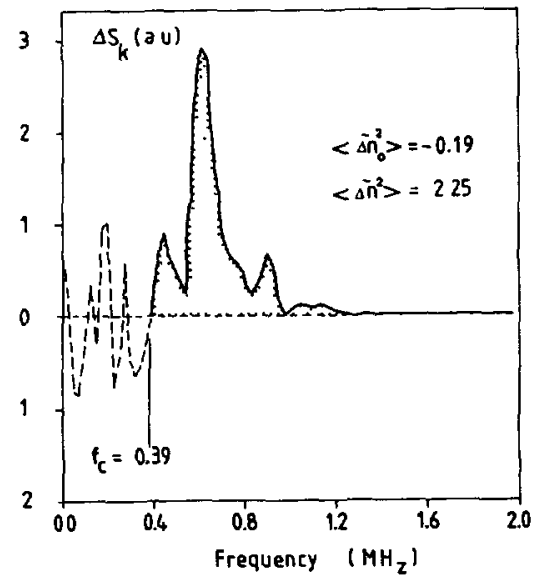

(c)

Fig. 6. - Determination of the "specific" fluctuations spectrum by substraction (central chord ; $\left.k=5 \mathrm{~cm}^{-1}\right)$. a) Average frequency spectrum $\left(S_{k}\right)_{\mathrm{av}}$ of the quasi-stationary turbulence $(\Delta f=24 \mathrm{kHz}$; $\left.\left\langle\tilde{n}^{2}\right\rangle_{\mathrm{av}}=\int\left(S_{k}\right)_{\mathrm{av}} \mathrm{d} f\right)$. b) Frequency spectrum $S_{k}$ of the total fluctuating signal during sawtooth collapse $(\Delta f=24 \mathrm{kHz})$. c) Frequency spectrum of the specific fluctuations $\Delta S_{k}=S_{k}-\left(S_{k}\right)_{\text {av }}$; the significant spectrum is for frequencies greater than $f_{\mathrm{c}} \cdot\left(\left\langle\tilde{n}_{0}^{2}\right\rangle=-0.19\right.$ corresponds to $\left.f<f_{\mathrm{c}}\right)$.

the shape and the amplitude of the spectrum prior and after the disruption are identical, showing that «specific» fluctuations and QS turbulence are two uncorrelated processes. A spectrum $S_{k}(\omega)$ is performed during the collapse phase (drop of the central temperature) (Fig. 6b). The difference $\Delta S_{k}(\omega)=S_{k}(\omega)-S_{k}(\omega)_{\mathrm{av}}$ is attributed to the associated sawtooth fluctuations (Fig. 6c). It essentially contains high frequency components. In the particular example shown in figure $6 \mathrm{c}, \Delta S_{k}(\omega)$ covers the frequency range $390 \mathrm{kHz} \leqslant f \leqslant 1200 \mathrm{kHz}$ and presents a maximum around $610 \mathrm{kHz}$. The amplitude for frequencies below $f_{\mathrm{c}}=390 \mathrm{kHz}$ retains an average value equal to zero. It exhibits the usual variations of the "quasi instantaneous " spectrum which are not significant. After an integration over frequency, we can compute the mean quadratic value $\left\langle\Delta \tilde{n}^{2}\right\rangle$ of the specific density fluctuations associated with the collapse. In this example, $\left\langle\Delta \tilde{n}^{2}\right\rangle$ largely exceeds $\left\langle\tilde{n}^{2}\right\rangle_{\mathrm{av}}\left(\left\langle\Delta \tilde{n}^{2}\right\rangle /\left\langle\tilde{n}^{2}\right\rangle_{\mathrm{av}} \approx 1.7\right)$, demonstrating the importance of the new phenomenon. 

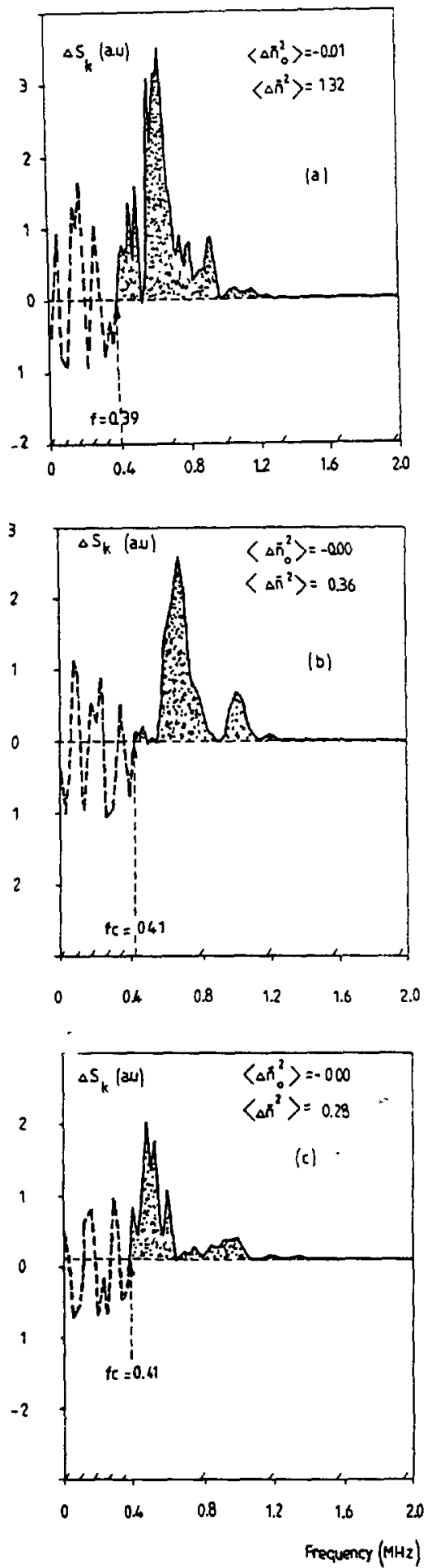

Fig. 7. - Three examples of frequency spectrum for the specific fluctuations during the collapse (central chord: $k=5 \mathrm{~cm}^{-1}$ ). 
For the same signal we show in figure 7a, a spectrum calculated over a period twice as long to give better resolution. The shape is roughly the same, but it shows the existence of some fine structure. We also present two other examples (Fig. $7 \mathrm{~b}, \mathrm{c}$ ) corresponding to different sawteeth in order to give an idea of the variability of this spectrum. A mean shape was obtained by superposition of several spectrum for different sawteeth and is shown in figure 5 of reference [50]. For higher values of the wave number $k$, the spectrum is displaced to higher frequencies and becomes smoother and enlarged (Fig. 4 of [50]). Globally, the relative width of the frequency spectrum is approximately one third $(\Delta \omega / \omega \approx 1 / 3)$.

5.1.2 Wave number spectrum. - The density fluctuation level $\left\langle\Delta \tilde{n}^{2}\right\rangle$ varies widely from one disruption to another, values being scattered from 1 to 10 for comparable disruptions. These wide variations will be understood in the next section 5.2. In spite of this large dispersion, we observe an average decay of $\left\langle\Delta \tilde{n}^{2}\right\rangle$ for increasing values of $k$ in the analysed domain $\left(5 \leqslant k \leqslant 17 \mathrm{~cm}^{-1}\right.$ ) (Fig. 8). In the double log scale, the mean trace is a line of slope $\approx-5 \pm 1$, showing a $k^{-5 \pm 1}$ variation law. This result is confirmed by several simultaneous measurements of two wave numbers $\left(5\right.$ and $17 \mathrm{~cm}^{-1}$ ). As compared to QS turbulence, the relative width in the decreasing part of the $k$ spectrum $S(k)=k^{-n}$ is two times narrower since $\Delta k / k \approx 1 / n$.

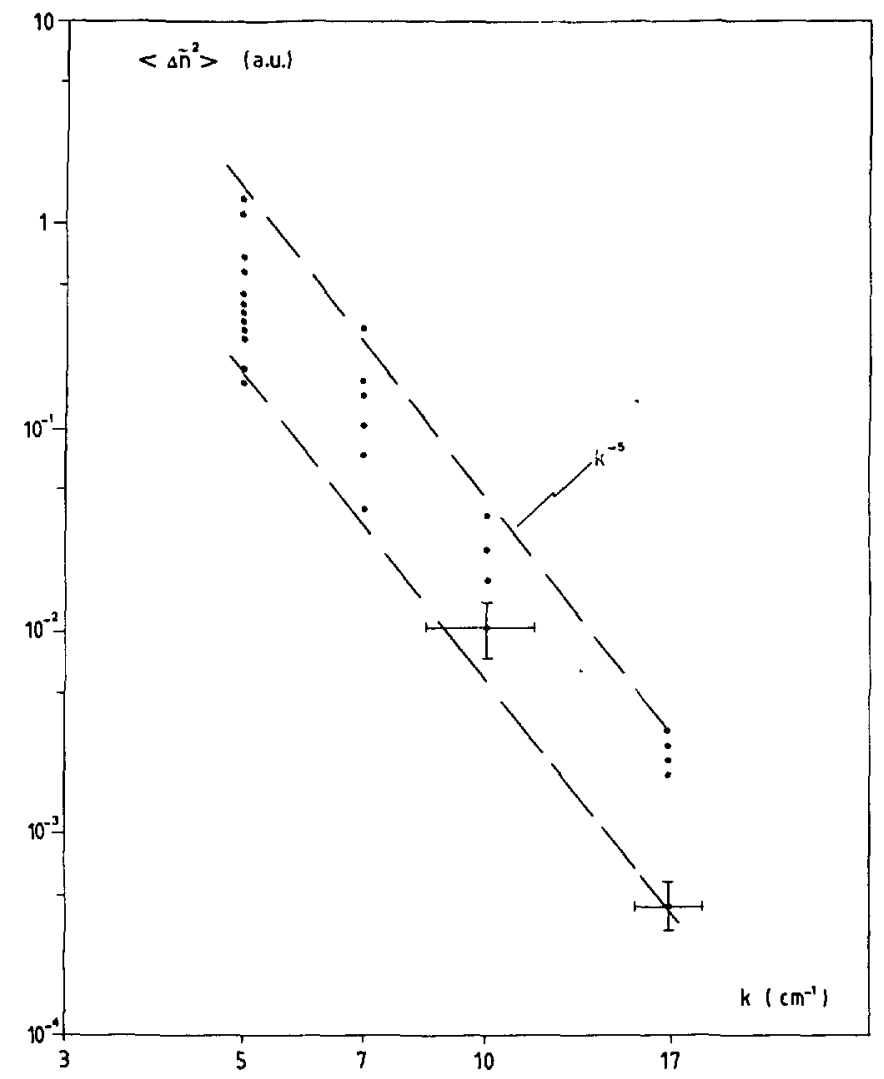

Fig. 8. - Wave number spectrum of the specific fluctuations (central chord).

5.1.3 Dispersion relation. - For a given wave number, the frequency spectrum is widely spread. However, we can trace a "pseudo" dispersion relation corresponding to the 
maximum of the frequency spectrum as a function of $k$. Figure $9 \mathrm{~b}$ shows such a dispersion curve. The dots mainly correspond to several disruptions although some of them correspond to the analysis. of two simultaneously measured $k$ numbers in the same disruption ( $k=5$ and $17 \mathrm{~cm}^{-1}$ for example). It is possible to define a mean phase velocity $V_{\mathrm{S}} \approx 8 \times 10^{3} \mathrm{~m} / \mathrm{s}$ which is significantly greater than the phase velocity of the quasi-stationary fluctuations $V_{\mathrm{QS}} \approx 2.3 \times 10^{3} \mathrm{~m} / \mathrm{s}$ shown on the same diagram. As already discussed [48] and deduced from figure 1 , the phase velocity of QS turbulence in the central region is inferred to be even smaller $\left(\approx 1.5 \times 10^{3} \mathrm{~m} / \mathrm{s}\right)$.

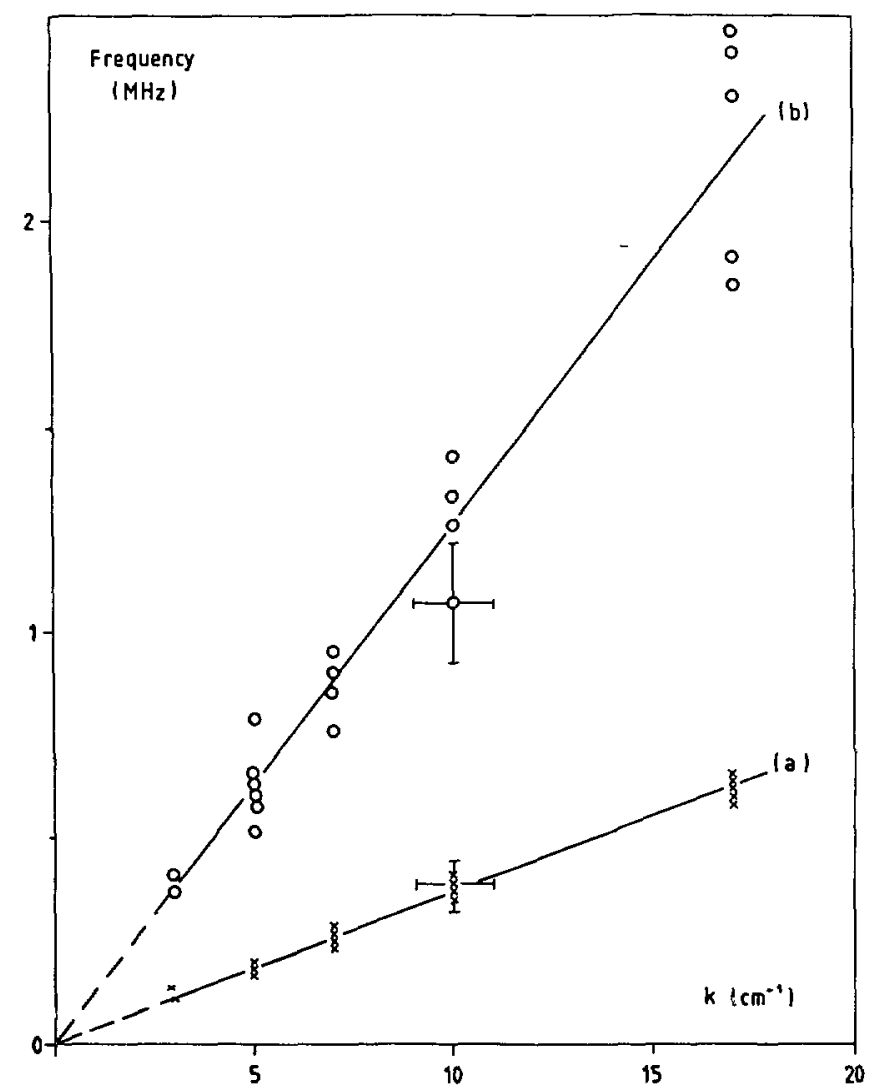

Fig. 9. - Dispersion curves (central chord). a) Quasi-stationary turbulence; mean phase velocity $v_{\mathrm{QS}}=2.3 \times 10^{3} \mathrm{~m} / \mathrm{s}$. b) Specific fluctuations; mean phase velocity $V_{\mathrm{s}}=(8 \pm 2) 10^{3} \mathrm{~m} / \mathrm{s}$.

5.2 SPATIAL LOCALIZATION. - For a fixed wave number we have analysed many disruptions, and plot in figure $10\left\langle\Delta \tilde{n}^{2}\right\rangle$ as a function of the position $X$ of the observation chord. The envelope of the various points gives a first estimate of the radial localization of the fluctuations. No signal is detected outside the $q=1$ surface and a depression of the amplitude is apparent around $X=0$, suggesting that the fluctuations are located somewhere inside the $q=1$ surface. A rough estimation of the width of the fluctuations domain can be deduced from the experiment and tends to be on the $2 \mathrm{~cm}$ scale. The spatial incertitude is mainly due to the laser beam width and the corresponding horizontal error bars are drawn on the figures. The incertitude in the specific fluctuations power is due to the Hanning window choice. It is 


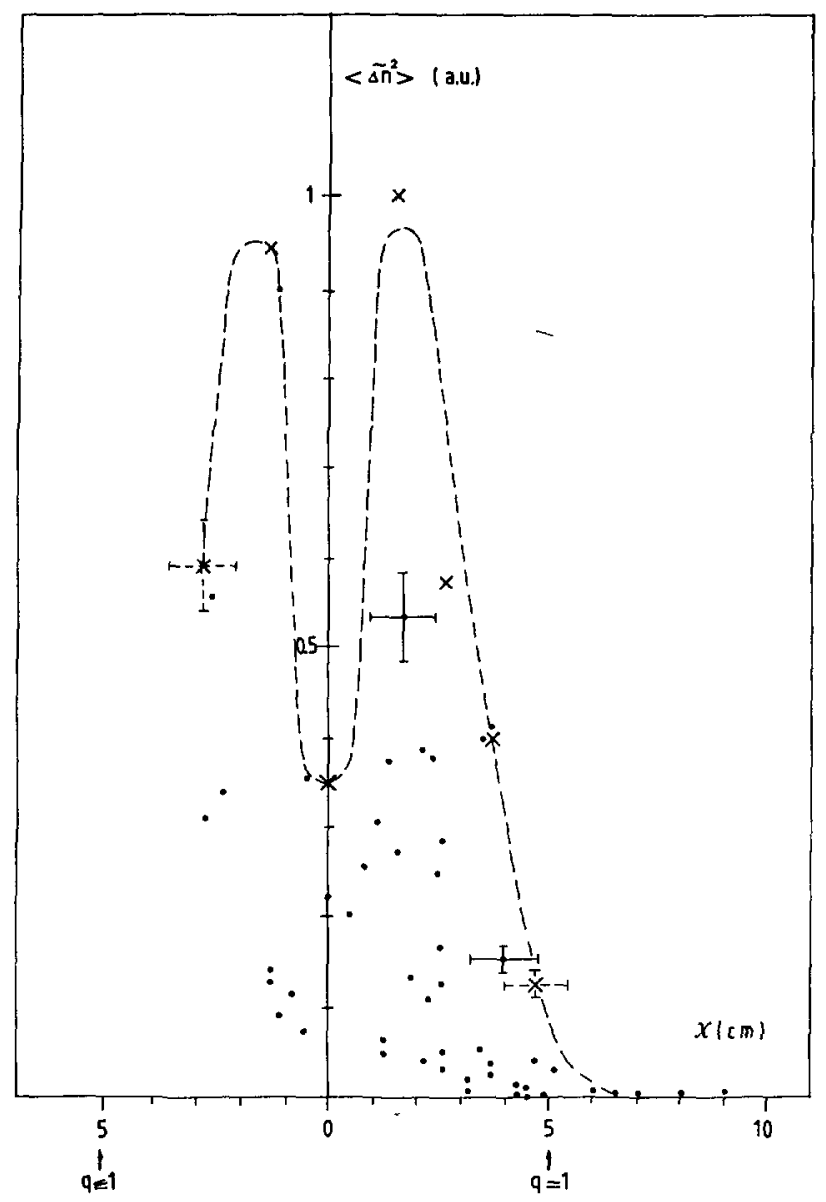

Fig. 10. - Radial scanning of the specific fluctuations for $k=7 \mathrm{~cm}^{-1}$. The different points corresponding to a same position are obtained from various similar crashes. Crosses are deduced from figure 12 and refer to azimuthal angle $\theta=\theta_{0}$. The dashed line represents the estimated radial variation for $\theta=\theta_{0}$.

estimated to be approximately ten per cent and is shown as vertical errors bars. The large dispersion of the points in figure 10 for a given value of $X$ suggests an azimuthal localization.

5.2.1 Azimuthal localization. - We shall separate the $r$ and $\theta$ variables, which comes to assume that the emiting cell has a bean shape, centered on a surface of radius $r_{0}$ with a characteristic radial extension $\Delta r_{\mathrm{C}}$ and a poloidal extension $\Delta \theta_{\mathrm{C}}$ (see Fig. 11). From the raw experimental points in figure 10 we choose $r_{0}=3 \mathrm{~cm}$. By following the $m=1$ mode observed on the soft X-ray signals just at the interior of the inversion radius $r \leq r_{1}$ (trace $b$ and a of Fig. 4), the azimuthal position of the cold side at the beginning of the second phase is called $\theta$. For one particular $X$ position of the scattering volume and one $k$ wave number, we analyze $\left\langle\Delta \tilde{n}^{2}\right\rangle$ as a function of $\left(\theta-\theta_{0}\right), \theta_{0}$ being the azimuthal angle of the $r_{0}$ circle intersection with the axis of the scattering volume. The data are symmetrical. Under these conditions, we choose $\left(\theta-\theta_{0}\right)^{2}$ as a new variable. Figures 12 and 13 show such a study for three $X$ positions. The problem of determining the angle is equivalent to determine the phase of a particular point on a sinusoidal curve. For doing this we use a time scale such that the 


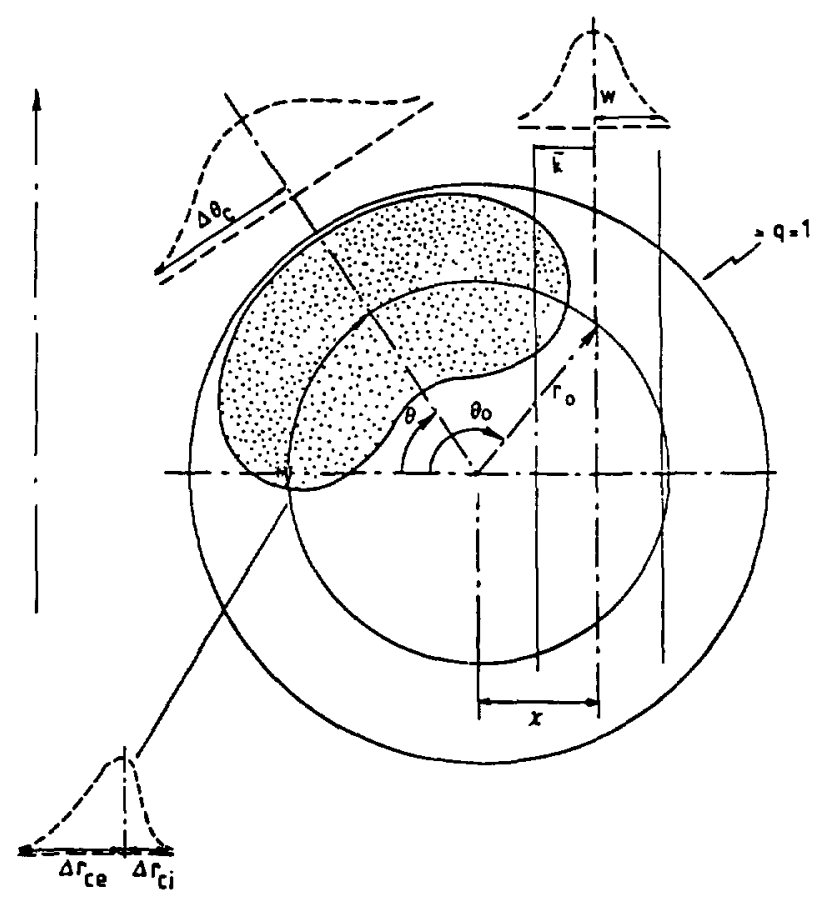

Fig. 11. - Main geometrical parameters. Schematic view of the "fluctuations cell " and of the scattering volume in a poloidal section.

signals show two or three oscillation periods. A careful marking of the successive maximum and minimum permits an estimation of the phase with an error less than $\pm 10^{\circ}$ to $15^{\circ}$. Data stemming from positive and negative values of $\left(\theta-\theta_{0}\right)$ are represented by different symbols. The amplitude depends drastically on $\left(\theta-\theta_{0}\right)^{2}$. It is maximum for $\theta=\theta_{0}$, with an experimentally observed half width of $\Delta \theta=60^{\circ} \pm 10^{\circ}$ for $k=5 \mathrm{~cm}^{-1}$ (Fig. 12) and $70^{\circ} \pm 10^{\circ}$ for $k=7 \mathrm{~cm}^{-1}$ (Fig. 13). From this analysis, we conclude that the strong fluctuations are azimuthally localized on the cold side.

5.2.2 Radial localization. - The maximum amplitude is observed when $\theta=\theta_{0}$, i.e. when the axis of the scattering volume coincides with the centre of the "fluctuations cell ». When no such experimental points exist, extrapolation of the line in figure 12 is used to estimate the maximum amplitude. These estimated maxima are reported as crosses in figure 10. They fall on an envelope (the dashed line). From this azimuthal and radial analysis, we conclude that the strong fluctuations are localized in a cell around a ring of radius $r_{0} \approx 2.5 \mathrm{~cm} \approx r_{1} / 2$.

5.2.3 High field side-low field side symmetry. - A careful analysis of the azimuthal law of variation of $\left\langle\Delta \tilde{n}^{2}\right\rangle$ for two $X$ positions symmetrical with respect to the magnetic axis have been performed (Fig. 13). Although the points gained from the outside chord quasi systematically exceed the inner ones, both signals are close and therefore are inconclusive as to the existence of a ballooning of the amplitude. The width of the localization cell, compared to the diameter of the scattering beam, is too small in TFR. This study should be undertaken on a bigger machine.

5.2.4 Numerical convolution. - The collected signal stemming from the various scattering volume elements is the result of a convolution in the Fourier space of the fluctuations cell 


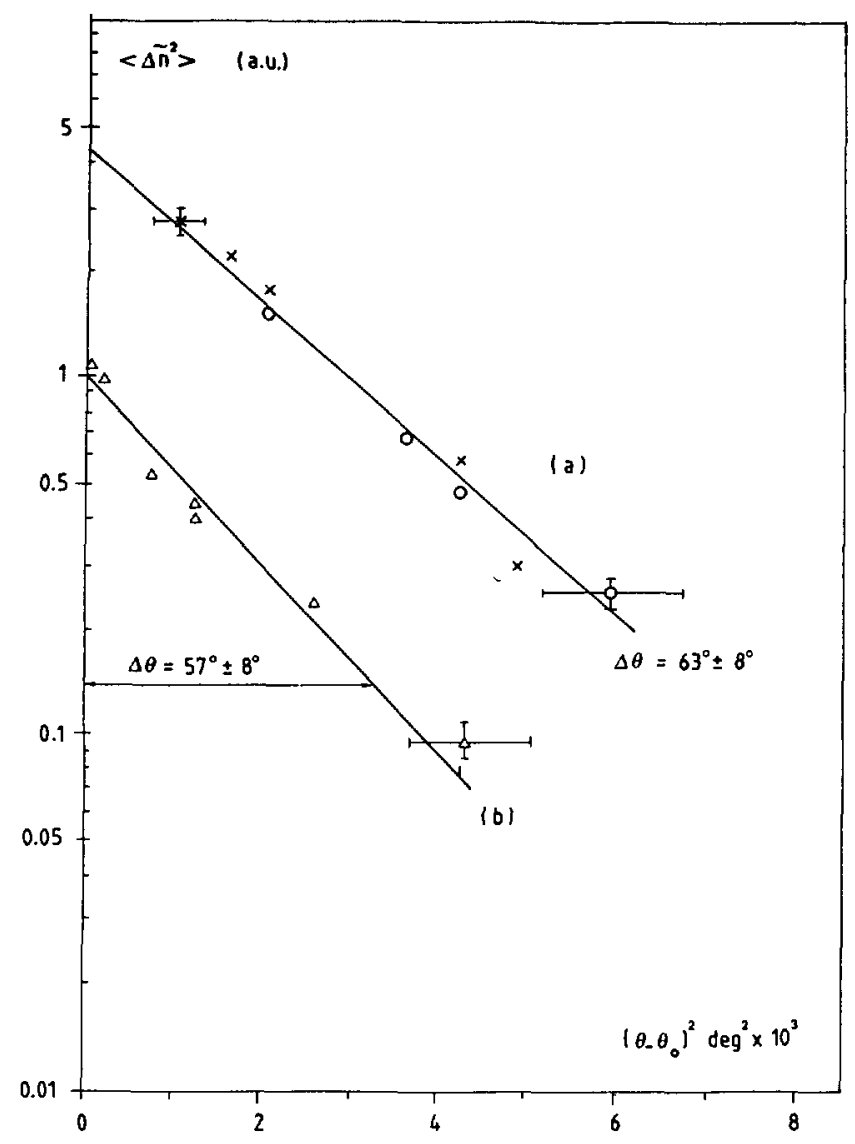

Fig. 12. - Azimuthal variation of the specific fluctuations for two different chords : $\left(k=5 \mathrm{~cm}^{-1}\right)$. a) $X=-1.5 \mathrm{~cm}$ (crosses for $\theta-\theta_{0}>0$ and open circles for $\theta-\theta_{0}<0$ ). b) $X=0 \mathrm{~cm}$ (diameter). The characteristic widths are defined at $1 / e^{2}$.

characteristics and of the probing laser beam. The object of this section is to estimate numerically the intrinsic properties of the fluctuations (spatial localization and $k$ spectral properties).

For a given wave number $\mathbf{k}$, the measured quadratic mean value of the fluctuating density associated to the sawtooth is

$$
\left\langle\Delta \tilde{n}^{2}\right\rangle=\alpha\left|\int \Delta \tilde{n}(\mathbf{r}) U(\mathbf{r}) \exp -(i \mathbf{k} \cdot \mathbf{r}) \mathrm{d} \mathbf{r}\right|^{2}=\alpha\left|\int \Delta \tilde{n}\left(\mathbf{k}^{\prime}\right) U\left(\mathbf{k}-\mathbf{k}^{\prime}\right) \mathrm{d} \mathbf{k}^{\prime}\right|^{2}
$$

where $\Delta \tilde{n}(\mathbf{r})$ is the local specific fluctuating density and $U(\mathbf{r})$ is the normalized local laser beam profile given by two projected Gaussian functions in a plane perpendicular to its direction of propagation i.e.

$$
U(\mathbf{r})=U_{0} \exp -\left(\left(X-X_{0}\right) / w_{0}\right)^{2} \exp -\left(Z / w_{0}\right)^{2}
$$

The analysis is limited to the poloidal plane (in the toroidal direction, the fluctuating density is assumed to have a characteristic length large compared to the beam diameter and will appear as a constant). 


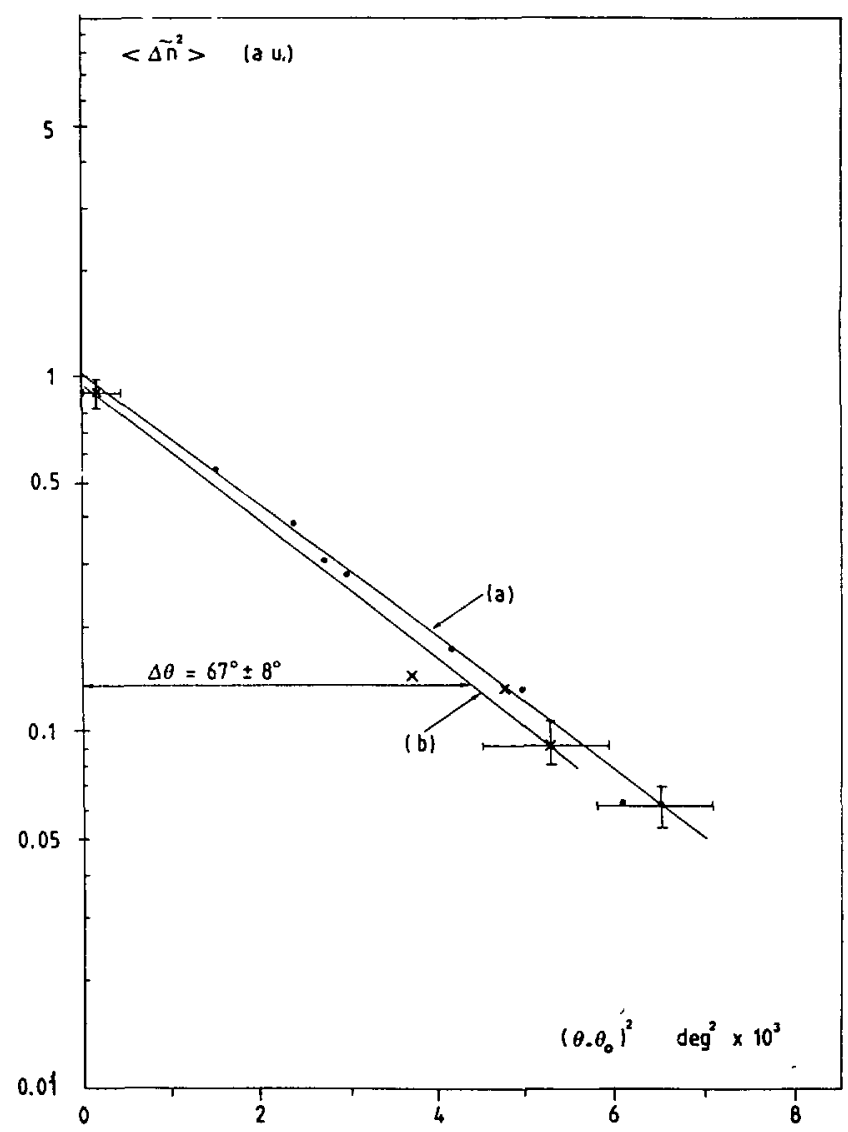

Fig. 13. - Azimuthal variation of the specific fluctuations for two symmetrical chords $\left(k=7 \mathrm{~cm}^{-1}\right)$ : a) $X=1.5 \mathrm{~cm}$; b) $X=-1.5 \mathrm{~cm}$.

If we assume that, on the scale of the beam size, the fluctuations have a locally isotropic spectrum in $k_{x}, k_{z}$ Fourier space, the computation is reduced to a convolution between the spatial amplitude distribution and the beam profile. We take for the fluctuations amplitude two Gaussian functions, in the radial $r$ and azimuthal $\theta$ directions, respectively centered around $r_{0}$ and $\theta_{0}$ i.e.

$$
A=A_{0} \exp -\left(\left(r-r_{0}\right) / \Delta r_{\mathrm{C}}\right)^{2} \exp -\left(\left(\theta-\theta_{0}\right) / \Delta \theta_{\mathrm{C}}\right)^{2}
$$

and determine numerically the values of $r_{0}, \Delta r_{C}$ and $\Delta \theta_{C}$ which give a good agreement with the experimental results.

A better agreement is obtained if we assume a radially asymmetric Gaussian repartition, with a characteristic width smaller for small radii. A good fit is found (Fig. 14) for a fluctuation cell defined by

$$
\begin{array}{rlrl}
r_{0} & =1.7 \mathrm{~cm} ; & \Delta \theta_{\mathrm{C}} & =1.2 \mathrm{rad} \\
\Delta r_{\mathrm{C}_{\mathrm{l}}} & =0.3 \mathrm{~cm} \text { for } r<r_{0} ; & \Delta r_{\mathrm{C} e}=2 \mathrm{~cm} \text { for } r>r_{0} .
\end{array}
$$

Several $k$ spectra have been tested. We note that a pure radial $k$ spectrum is inconsistent with the observations and can be definitively discarded. Conversely, a purely azimuthal $k$ spectrum can account for the experiments with $r_{0} \approx 3 \mathrm{~cm}$ and $\Delta r_{\mathrm{Cl}} \approx \Delta r_{\mathrm{C} e} \approx 2 \mathrm{~cm}$. 

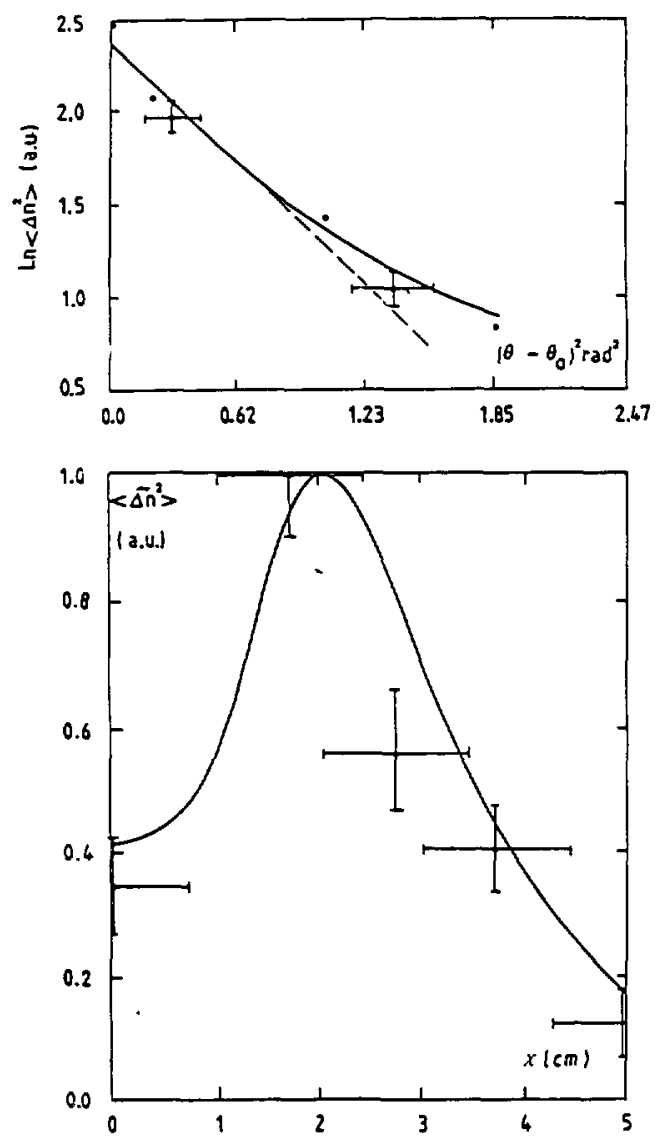

Fig. 14. - Comparison between numerical convolution and experiment $\left(k=7 \mathrm{~cm}^{-1}\right)$. Top : azimuthal specific density fluctuations variations. Bottom: radial profile. Cell parameters are $: r_{0}=1.7 \mathrm{~cm}$, $\Delta \theta_{\mathrm{C}}=1.2 \mathrm{rad}, \Delta r_{\mathrm{C}}=0.3 \mathrm{~cm}$ for $r<r_{0} ; \Delta r_{\mathrm{Ce}}=2 \mathrm{~cm}$ for $r>r_{0}$.

Consequently, we conclude that an approximately isotropic spectrum is present, on which a partially azimuthal contribution could be superimposed. We insist that the «fluctuations cell " as drawn in figures 11 and 17 is not a domain inside which fluctuations are restricted, but simply a sketch of the place where the amplitude is stronger.

5.3 Power LEVEL. - The amplitude of the specific fluctuations will not be evaluated in absolute terms, but rather compared with the local quasi-stationary fluctuations present in the central region. The essential element in this discussion is the fact that the measured fluctuations come from volume elements positioned along a chord (here a diameter) through the plasma. In order to make our estimation, the radial domain is divided in three regions : a central region (symbol c) for $r / a<0.4$, a gradient region (symbol G) for $0.4<r / a<0.9$ and an edge region (symbol $\mathrm{E}$ ) for $0.9<r / a<1.1$.

In these conditions, and by assuming that the fluctuations from different regions are statistically not correlated, the total Q.S. collected fluctuations quadratic mean value is :

$$
\left\langle\tilde{n}^{2}\right\rangle=\left\langle\left(\int_{\mathrm{E}} \mathrm{d} \ell \tilde{n}\right)^{2}\right\rangle+\left\langle\left(\int_{\mathrm{G}} \mathrm{d} \boldsymbol{\ell} \tilde{n}\right)^{2}\right\rangle+\left\langle\left(\int_{\mathrm{C}} \mathrm{d} \boldsymbol{\ell} \tilde{n}\right)^{2}\right\rangle
$$


or $\left\langle\tilde{n}^{2}\right\rangle=\left\langle\tilde{n}^{2}\right\rangle_{\mathrm{E}}+\left\langle\tilde{n}^{2}\right\rangle_{\mathrm{G}}+\left\langle\tilde{n}^{2}\right\rangle_{\mathrm{C}}$ if the short notation $\left\langle\tilde{n}^{2}\right\rangle$ is used in place of $\left\langle\left(\int \mathrm{d} \ell \tilde{n}\right)^{2}\right\rangle$

Admitting that the radial variation of the relative density fluctuations in a Tokamak is well represented by the information gathered by Liewer in figure 8 in her review article [56] we have the approximate relation: $(\tilde{n} / n)_{\mathrm{E}} /(\tilde{n} / n)_{\mathrm{C}} \approx 30$ to 50 . Taking into account the observed density profile such that $n_{\mathrm{C}} / n_{\mathrm{E}} \approx 6$ and the relative length of the concerned regions, we get : $\left\langle\tilde{n}^{2}\right\rangle_{\mathrm{E}} \approx 10\left\langle\tilde{n}^{2}\right\rangle_{\mathrm{C}}$. A similar estimation for the gradient and edge regions gives: $\left\langle\tilde{n}^{2}\right\rangle_{\mathrm{G}} \approx\left\langle\tilde{n}^{2}\right\rangle_{\mathrm{E}}$. Thus for the total Q.S. fluctuations we have : $\left\langle\tilde{n}^{2}\right\rangle \approx 20\left\langle\tilde{n}^{2}\right\rangle_{\mathrm{C}^{*}}$.

For the example shown in figure 6 the ratio between the radially integrated specific $\left\langle\Delta \tilde{n}^{2}\right\rangle$ and the total Q.S. density fluctuations is such that : $\left\langle\Delta \tilde{n}^{2}\right\rangle /\left\langle\tilde{n}^{2}\right\rangle \approx 1.7$. For a well centered cell $\left(\theta=\theta_{0}\right)$ the graph of figure 12 gives by extrapolation : $\left\langle\Delta \tilde{n}^{2}\right\rangle /\left\langle\tilde{n}^{2}\right\rangle \approx 2$ and so $\left\langle\Delta \tilde{n}^{2}\right\rangle /\left\langle\tilde{n}^{2}\right\rangle_{\mathrm{C}} \approx 40$.

This ratio applies to fluctuating densities integrated on appropriated volumes of scattering. For the specific fluctuating density it is the cell volume included in the diagnosing beam. Taking into account the dimensions and the position of the cell we find that this volume is approximately one half of the scattering volume for the Q.S. fluctuations in the central region. Then the evaluation of the ratio between the local values of the specific and Q.S. fluctuations gives :

$$
\Delta \tilde{n}^{2} / \tilde{n}_{\mathrm{C}}^{2} \approx 100 \text { to } 200 \text {. }
$$

The fact that the specific fluctuations appear to be maximum for $k<5 \mathrm{~cm}^{-1}$ whereas QS turbulence is maximum for $k \approx 5 \mathrm{~cm}^{-1}$ could increase this ratio a little.

Assuming a central QS relative density fluctuation equal to 1 to $3 \times 10^{-3}$, the specific fluctuations are estimated to reach the value: $(\Delta \tilde{n} / n)_{\mathrm{C}} \approx 2$ to $3 \times 10^{-2}$

We conclude that the specific fluctuations represent a strong phenomenon which appears a good candidate for explain the strong increase of the transport during the collapse. Indeed, we estimate the heat duffusivity during the collapse as $\chi_{\text {collapse }} \approx r_{1}^{2} / 4 \tau_{\text {collapse, }}$, where $r_{1}=5 \mathrm{~cm}$, and $\tau_{\text {collapse }} \approx 20 \mu \mathrm{s}$, then :

$$
\chi_{\text {collapse }} \approx 3 \times 10^{5} \mathrm{~cm}^{2} / \mathrm{s} \approx 100 x_{\mathrm{Qs}} .
$$

\section{Heat flux asymmetry.}

During the collapse, the heat flux ouside the $q=1$ surface is not azimuthally symmetrical. This asymmetry can be seen on the SX signals. For example in figure 4 the heat flux appears "sooner and faster" on chord (a) compared to chord (e). On the compass card rose of figure 15 , each point represents the azimuthal direction $\theta$ of the hot side in the plasma cross section observed by the soft X-ray detectors. For each observed sawtooth we note at the corresponding point, the direction of the "sooner and faster " heat flux by an arrow with an amplitude proportional to the sharpness of the asymmetry. We conclude that when the hot side of the precursor, whose rotation is extrapolated to the beginning of the collapse, is situated in the $\theta$ direction indicated on the figure by a cross (on the transition diameter), the "sooner and faster" heat flux direction is $\theta=270^{\circ}$. Therefore assuming an unmodified rotation velocity of the hot core during the kink, the heat flux appears to be directed $3 \pi / 4$ away of the hot side, i.e. apparently at one corner of the fluctuations cell.

This result appears somewhat surprising at first glance. However, if we apply Andreoletti's 


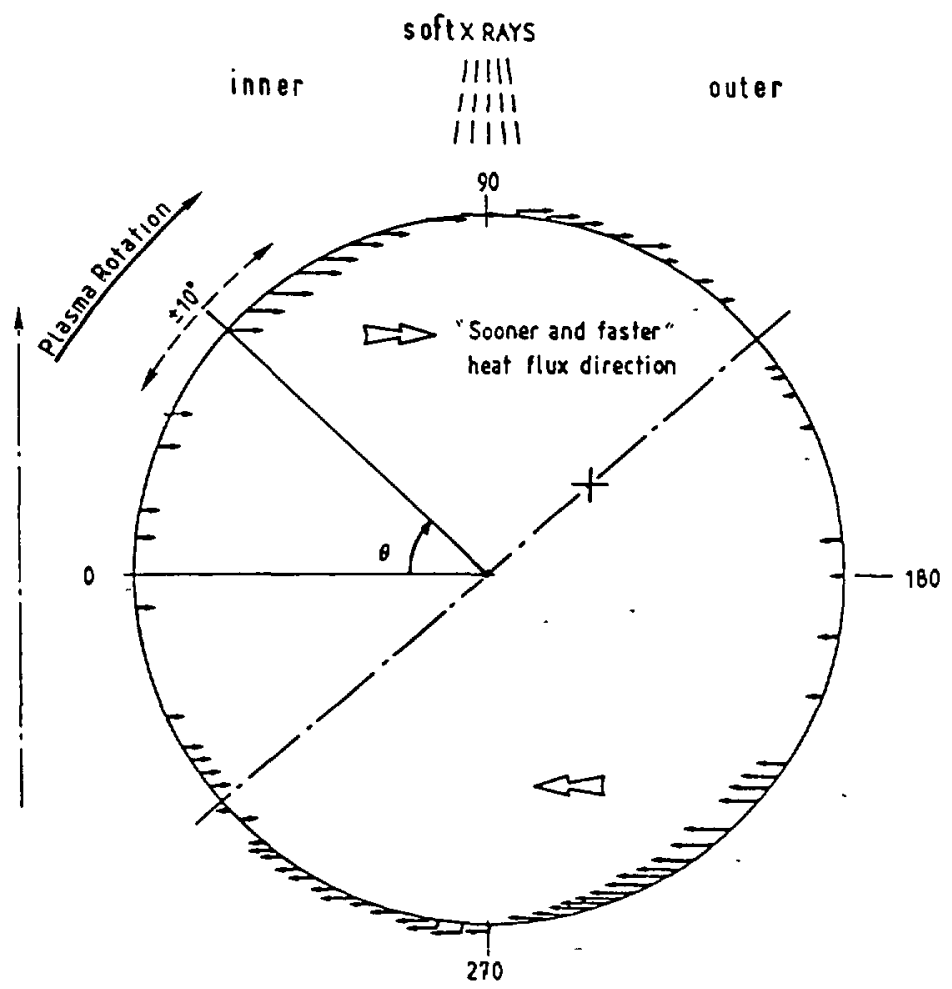

Fig. 15. - Direction of the "sooner and faster » heat flux during the collapse (soft X-ray analysis). $\theta$ refers to the azimuthal hot core position, at the beginning of the collapse.

model (see next chapter), the plasma rotation could be modified by the turbulent kink. The rotation of the edge part $\left(r \leq r_{1}\right)$ slows down whereas the one of the more central part speeds up, producing a twisted state. A subsidary observation tends to confirm the existence and the large value of the twisting. We again draw, from the SX signals, a compass card rose indicating the apparent direction of the fast kink displacement with respect to the azimuthal position of the precursor asymmetry (Fig. 16). The angle $\theta$ is the azimuthal position of the precursor hot side at the beginning of the fast kink. The arrow length is proportional to the observed amplitude of the fast displacement. When the hot side is situated in the $\theta$ direction indicated by a cross, the fast displacement direction is $\theta=270^{\circ}$. The result is that the fast kink is seen as if it was directed at $\pi / 2$ in advance with respect to the precursor. This can be explained by a composition of the kink displacement and an accelerated rotation of the hot core $\left(\sim 50\right.$ to $\left.60^{\circ}\right)$. The estimated twisting angle is large on TFR $(\sim \pi / 4)$ because the plasma rotation is fast. Under these conditions, the azimuthal direction of the "sooner and faster " heat flux would coincide with the central position of the fluctuations cell (Fig. 17). On large machines the plasma rotation is much slower and thus twisting is much less pronounced but visible (see for example Fig. 22 of [46]).

The coincidence between the direction of the « sooner and faster " outwards heat flux, and the centre of the fluctuations cell is a strong experimental element in favour of the increased diffusivity being due to these fluctuations. Measurements performed on a large machine having a slowly rotating plasma would give a more direct access to this information without being confused by an additional rotation. 


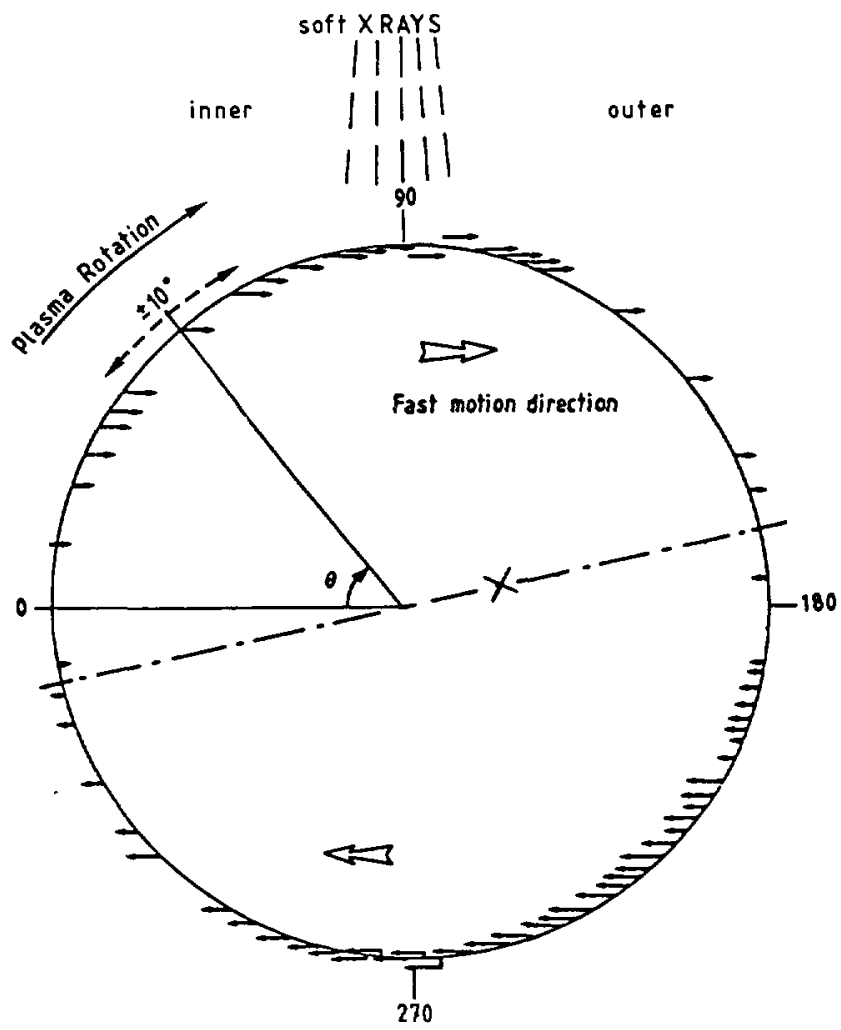

Fig. 16. - Direction of the hot area fast displacement during the second phase (soft X-ray analysis). $\theta$ refers to the azimuthal hot core position, at the beginning of the fast kink.

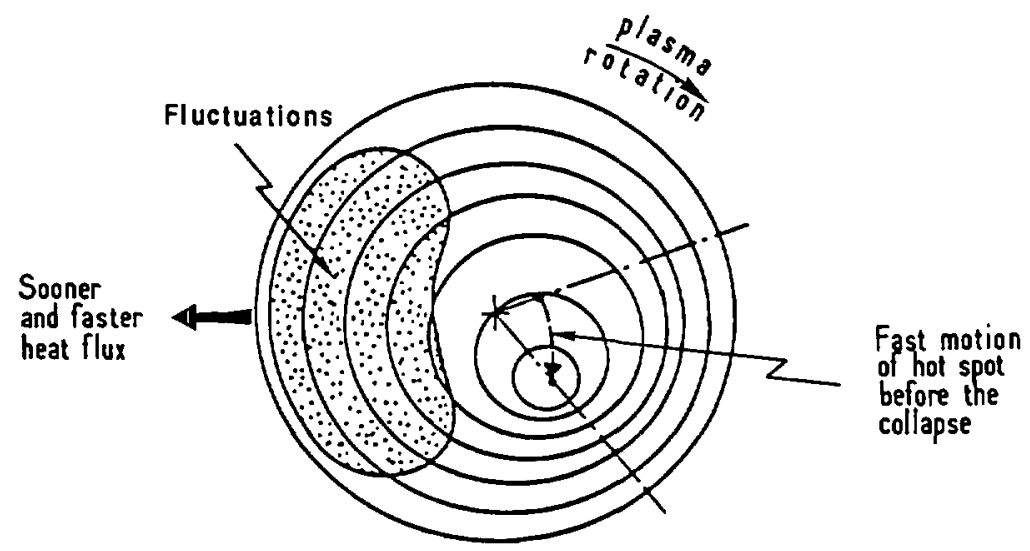

Fig. 17. - Summary of the geometrical situation during the collapse.

\section{Comparison with the turbulent models.}

7.1 NASCENT EDDY STRUCTURED TURBULENCE. - Before undertaking a comparison between our observations and predictions from the turbulent models, we shall discuss in what restrictive sense the observed specific fluctuations can have to do with turbulence. 
In the evolution, in space or in time, of any fluid from a stable laminar or equilibrium situation to a state of fully developed turbulence, one can distinguish three stages. The first two are dominated by the production process. In the linear stage the instability gives rise to small amplitude oscillations with narrow wave number and frequency spectra. During the second non linear stage, the motion reaches a large saturated amplitude with a rather regular spatial structure, and with somewhat enlarged spectra. In the last stage, the production process becomes a small effect just compensating the energy losses due to dissipation, and the system is dominated by propagation and transport in real space, and in the frequency and wave number spaces. The spectra are much enlarged and the spatial structure is irregular. The system asymptotically tends to a state of fully developed turbulence for which a statistical description is relevant.

Since the observed specific fluctuations are situated in the same space and time domain than the production process itself, there is no possibility to reach an asymptotic state of fully developed statistical turbulence. The facts that the frequency and wave number spectra are moderately broad (a factor of two or three less than for QS turbulence) and the amplitude is large suggest that these fluctuations correspond to the intermediate state of " nascent structured turbulence $»$.

In the magnetodrift turbulent model the fluctuations are spatially structured by the high velocity shear of the return flux due to the kink instability. This convective motion is developing during the kink phase, becoming faster during the collapse and reversing during the last phase. The return flux of these convective cells forms two adjacent vortex sheets of opposite signs and the effect of the inertial instability is to split up these sheets in a series of eddies of opposite signs drifting in the mean return stream.

A recently published experimental result [47] obtained from five arrays of soft X-ray detectors tends to support this picture. The emissivity map shown in figure 18 is a reproduction of figure $3 \mathrm{c}$ of this reference. The wavy lines are apparently inside but not far from the $q=1$ surface. The finite extent of the oscillation domain and the divergence free character of the velocity field imply the existence of eddies. An outline of their central part has been drawn on the figure.

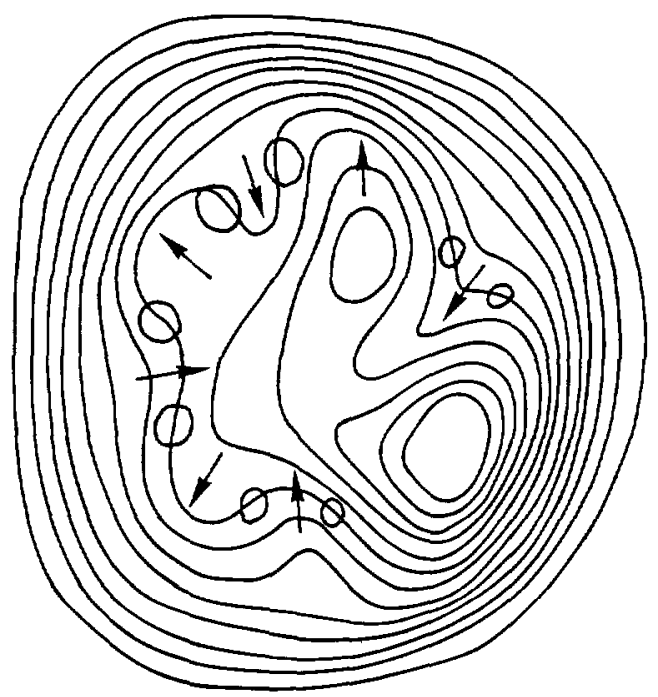

Fig. 18. - Soft X-ray emissivity map during the fast helical displacement in the Tokamak of Varennes [41]. Outline of the suggested velocity field has been superimposed. 
7.2 COMPARISON WITH THE RESISTIVE mhd TURBULENT MODEL. - In this model [29, 30, 31] the sawtooth crash is initiated by a resistive tearing instability developing into an island. At some point in the evolution, a singular current density layer appears on the separatrix, corresponding to the cancellation of the flux lines angle at the $X$ point. This current sheet is unstable to high $\mathrm{m}$ resistive modes and a turbulent region appears and propagates from the two branches of the separatrix to the inner and outer regions. Flattening of the electron temperature and current density profiles is expected in these domains. The island itself is unchanged. Then the possible residual hot core comes back to axisymmetry. This early proposition was a tentative to remedy the slowness of the full reconnection model by propagating the singular current layer through the plasma. But the resulting scenario disagrees with several aspects of the sawtooth phenomenon. We first notice that precursorless sawteeth are observed and the island, if any, is thus undetectably small. If we nevertheless suppose that an island is formed, we note the absence in the model of a fast displacement phase between the slow resistive island formation and the turbulent collapse. Then the turbulence generated by the current sheet is evenly distributed in the $\theta$ direction, and is situated partly inside and partly outside the $q=1$ surface. In contrast the observed fluctuations reported here are azimuthaly localized and do not exist for $r>r_{1}$. Cold bubble formation and crescent distortion of the hot core observed on X-ray tomography maps during the collapse are not explained by the model. Also considering the outgoing heat flux, any asymmetry in the model would be oriented in favour of the $X$ point where the two turbulent domains are in contact, rather than in the wake of the displacement.

7.3 COMPARISON WITH THE TWO FLUIDS MAGNETODRIFT TURBULENT MODEL. - A qualitative turbulent model has been proposed by Andreoletti as a tentative explanation of the sawtooth mechanism [33, 34]. Here, we propose only to discuss the main elements correlated to the above mentioned experimental data.

Nevertheless, for clarity we shall recall the main arguments sustaining this model. As discussed in the introduction internal relaxation phenomenon appears impossible in the frame of ideal mhd, mainly because of flux preservation. Some parallel electric field is necessary and the proposed model rely on the $\nabla p_{\mathrm{e}} / e n_{\mathrm{e}}$ term of Ohm's law. This term can induce magnetic field only if the plasma develops some "baroclinity " (non zero inclinaison of constant pressure surfaces with respect to constant density surfaces). Formation of baroclinity can result from the strong disparity of the electron and ion masses, if a sufficiently short wavelengh appears along magnetic field lines. In a low $\beta$ plasma the parallel wavenumber $k_{\|}$is not a free parameter due to the constraint of minimizing the magnetic energy perturbation. It is related to the local magnetic shear $\hat{s}_{*}$, and in the case of a quasi isotropic perpendicular wavenumber $k_{\perp}$ spectrum we have $k_{\|} \approx \pi \hat{s}_{*} / q R$. In a configuration with sufficient large scale local shear asymmetry, the variation of $k_{\|}$along a magnetic line can be such that motions exist that agree with mhd dynamics in the low shear part and with drift wave dynamics in the high shear part. These motions have been called " magnetodrift». The central mechanism in the model is the interplay between the kink instability that develops local magnetic shear asymmetry and drives magnetodrift (md) oscillations, and these md fluctuations that breaks magnetic surfaces and allows the development of the convective motion.

Four phases are defined : resistive kink, turbulent kink, collapse and return. We shall now successively compare the predictions of the model for each of the four phases, with our observations of soft X-ray and of density fluctuations.

7.3.1 The resistive kink. - Just after the preceding relaxation the pressure gradient inside $r_{1}$ is small, and according to the model the shear parameter $\hat{s}_{1}$ at $r=r_{1}$ is also small. During 
the regeneration period they increase and the threshold for the resistive kink instability can be reached. The growth rate for the resistive kink, taking into account diamagnetic and rotation effects [10] gives $\gamma \approx 4 \times 10^{3} \mathrm{~s}^{-1}$ This is a little faster than the observed evolution of the precursor oscillation whose growth time is $\tau \approx 300$ to $400 \mu \mathrm{s}$.

7.3.2 The turbulent kink. - According to the model, the asymmetry of the local magnetic shear produced in the resistive sheet, and the high velocity shear of the return flux give rise to inertially driven md turbulence. This turbulence triggers a fast transition from resistive kink to quasi-mhd kink and allows its catastrophic increase. The absence of precursor oscillations generally observed on large tokamaks is understood in the following way: when the $q=1$ radius $r_{1}$ is large, the Reynolds number for the return velocity stream in the resistive layer is large, and so the transition from resistive kink to mhd kink due to md turbulence occurs soon. Since the duration of the resistive kink phase is short, and also rotation of large plasmas is slow, precursor oscillations are not seen.

The radial velocity of the fast kink observed during the TFR sawteeth is approximately $V_{\mathrm{r}} \approx 2 \times 10^{5} \mathrm{~cm} / \mathrm{s}$. The return flux velocity for smooth convective cells as observed on JET tomography pictures [43] is estimated to be : $V_{\theta} \approx 2 V_{\mathrm{r}} \approx 4 \times 10^{5} \mathrm{~cm} / \mathrm{s}$. The typical frequency of the inertially driven turbulence will be such that $\omega \approx k V_{\theta}$. For $k=5 \mathrm{~cm}^{-1}$ this gives $f=300 \mathrm{kHz}$, a value that could agree with our experimental estimations given in section 4 .

The velocity fluctuations are expected to be (in similarity with hydrodynamics) about half an order of magnitude smaller than the return stream velocity: $\delta V \approx 1 / 3 V_{\theta} \approx$ $4 / 3 \times 10^{5} \mathrm{~cm} / \mathrm{s}$. From the relation :

$$
\delta V \approx k \delta \phi / B \approx k \rho_{1} V_{\mathrm{i}}\left(T_{\mathrm{e}} / T_{\mathrm{j}}\right)\left(e \delta \phi / T_{\mathrm{e}}\right)
$$

where $k$ is $k_{\perp}, \delta \phi$ is the scalar potential fluctuation, and $V_{1}, \rho_{i}$ are the ion thermal velocity and the ion mean Larmor radius, we get for the density fluctuations the rough estimate:

$$
\tilde{n} / n \approx\left(T_{\mathrm{i}} / T_{\mathrm{e}}\right)\left(1 / k \rho_{\mathrm{i}}\right)\left(\delta V / V_{\mathrm{i}}\right) \approx 6 \times 10^{-3} \approx 3(\tilde{n} / n)_{\mathrm{QS}}
$$

and so

$$
\left\langle\tilde{n}^{2}\right\rangle_{\mathrm{knk}} \approx 10\left\langle\tilde{n}^{2}\right\rangle_{\mathrm{QS}}
$$

Our experimental estimate for the non hidden part $(400 \mathrm{kHz}<f<500 \mathrm{kHz})$ is :

$$
\left\langle\tilde{n}^{2}\right\rangle_{\mathrm{kank}} \approx 1 / 30\left\langle\tilde{n}^{2}\right\rangle_{\mathrm{collapse}} \approx(1 / 30)(100 \text { to } 200)\left\langle\tilde{n}^{2}\right\rangle_{\mathrm{QS}} \approx 5\left\langle\tilde{n}^{2}\right\rangle_{\mathrm{QS}} .
$$

So an agreement between both estimations of $\left\langle\tilde{n}^{2}\right\rangle_{\mathrm{knk}}$ is possible if we suppose that fluctuations power in the generally masked part $(200 \mathrm{kHz}<f<400 \mathrm{kHz})$ is of the same order as the one of the visible part.

The plasma rotation will be modified by the turbulent kink. The model includes the breaking of the magnetic surfaces in the annular md turbulence domain. Due to the resulting preferential escape of the light electronic gas from this zone, a local positive charge density will appear among the generally observed [57] negative potential and negative charge density of the plasma. The corresponding local modification of curvature of the potential profile will result in some flattening in the $r \approx r_{1}$ region and some steepening in the more central domain. The consequence on the $\mathbf{E} \times\left(\mathbf{B} / B^{2}\right)$ rotation is a slowing down in the $r \approx r_{1}$ region and an enhancement of the central core rotation, giving rise to some twisting of the plasma.

7.3.3 The collapse. - The collapse of the temperature and density profiles is due, in the 
model, to a strong burst of md turbulence. The occurrence of this burst is related to some threshold in the helical asymmetry of the local magnetic shear. This turbulence is mainly located in the wake of the kink motion, and could explain the cold bubble formation and the crescent distortion of the shifted hot core [43]..

As we have seen in the discussion of section 6 , the azimuthal positions of respectively the observed fluctuations cell and the " sooner and faster » heat flux are not precisely opposite to the hot spot position during the collapse. They present a phase lag of approximately $\pi / 4$ relative to the direction of plasma rotation, and the model suggests an explanation of this angular discrepancy by the twisting effect (Fig. 17).

The md turbulent motions are well represented by mhd dynamics in the wake, and by drift wave dynamics on the side and in front of the kink displacement. The local magnetic shear parameter $\hat{s}_{*}$ on the side being approximately equal to the initial global shear $\hat{s}$ we have for the frequency the estimate

$$
\omega \approx \alpha\left(V_{\mathrm{l}} V_{\mathrm{e}}\right)^{1 / 2} k_{\|} \approx \alpha\left(V_{\mathrm{l}} V_{\mathrm{e}}\right)^{1 / 2}(\pi \hat{s} / q R)
$$

with $1<\alpha \ll(M / m)^{1 / 4}$, that is $\alpha \approx(M / m)^{1 / 8}$, $\alpha$ being a parameter representing the expected frequency shift towards the mhd domain $\omega \gg k_{\|} V_{\mathrm{e}}$. In the proposed model, the magnetic shear $\hat{s}$ increases during the turbulent kink and we assume that, at the end of this phase, its value has become similar to the one of a regular current density profile. This gives us $\hat{s} \approx 0.25$ for $r \leq r_{1} \approx a / 3$. Thus we get :

$$
f \approx(2.8 / 2 \pi)\left(2 \times 10^{7} \mathrm{~cm} / \mathrm{s} \times 1.5 \times 10^{9} \mathrm{~cm} / \mathrm{s}\right)^{1 / 2}(0.25 \pi / 100 \mathrm{~cm}) \approx 0.6 \mathrm{MHz}
$$

in good agreement with our observations.

The level of the specific fluctuations during the collapse as observed experimentally has been estimated (Sect. 5) to reach the value $\Delta \tilde{n} / n \approx 2$ to $3 \times 10^{-2}$. This is more than the usual estimate from comparison of the perturbed and initial gradients $\tilde{n} / n \approx 1 / k r_{n} \approx 10^{-2}$ and it is one third to one half of the initial density bump $\Delta n / n \approx\left(r_{1} / a\right)^{2} \approx 6 \times 10^{-2}$ The numbers suggest that the observed strong fluctuations are in an extreme nonlinear regime in which the quadratic term $\delta \mathrm{V} \cdot \nabla n$ is of the same order as the linear ones. This corresponds to $\delta V \approx \omega / k$ and gives the estimate

$$
(\tilde{n} / n)_{\mathrm{NL}} \approx\left(T_{\mathrm{i}} / T_{\mathrm{e}}\right)\left(1 / k_{\perp} \rho_{1}\right)(\omega / k) / V_{\mathrm{i}} \approx 3 \times 10^{-2}
$$

We have seen (Sect. 5.1.2) that between $k=5 \mathrm{~cm}^{-1}$ and $k=17 \mathrm{~cm}^{-1}$ specific fluctuations are strongly decreasing $\sim k^{-5}$ We can then guess that the maximum of the wave number spectrum corresponds to a $k$ value less than $5 \mathrm{~cm}^{-1}$, say 4 or $3 \mathrm{~cm}^{-1}$ As to the large scale kink, the known crescent deformation includes harmonics at least $m=2, m=3$ which correspond to $k \approx 1 \mathrm{~cm}^{-1}$. We observe that the unknown interval appears rather small. This remark, and the fact that the estimated density fluctuation is a large fraction $(1 / 3$ to $1 / 2)$ of the initial density bump, suggest that there may be no gap between the large scale and small scale motions, but we have no direct proof of that. However the wavy emissivity lines in figure 18 (from the recent paper [47]) could represent a first experimental observation of the intermediate scale motion.

Coupling between the small scale and the large scale parts of the motion is expressed by equating the mean phase velocity of the magnetodrift oscillations $\omega / k \approx k_{\|} \alpha\left(V_{\mathrm{i}} V_{\mathrm{e}}\right)^{1 / 2} /\left(2 \rho_{\mathrm{i}}\right)^{-1}$ to the return velocity of the convective kink motion $V_{\theta} \approx 2 V_{\mathrm{r}} \approx 2 r_{1} / \tau_{\text {collapse. }}$. This coupling condition determines the characteristic time for the collapse

$$
\tau \approx R r_{1}\left[\pi \hat{s} \alpha V_{\mathrm{e}}^{1 / 2} V_{\mathrm{i}}^{1 / 2} \rho_{1}\right]_{r=r_{1}}
$$


For TFR we get $\tau_{\text {collapse }} \approx 15 \mu$ in good agreement with the observed duration of the collapse phase.

7.3.4 The return. - When the pressure gradient has vanished md turbulence dies away. According to the model, the return of the magnetic structure to a quasi-circular axisymmetrical shape must be fast and will produce a new burst of md turbulence similar to the one during the turbulent kink phase, resulting in the formation of an annular plateau $q \approx 1$ in the $q(r)$ profile. Our observations have shown that in fact specific density fluctuations are similar during the kink and return phases.

Depending on the flatness of the central current density profile (fast current rise, impurities accumulation, non central additional heating...) there can be variations in the evolution of the magnetic structure with a much more pronounced distortion and erosion of the shifted core, resulting in no return. In this case, the corresponding full reconnection will contribute to the maintenance of the central current flatness. But these variations in the evolution are part of the model itself and will be argued elsewhere.

\section{Conclusion.}

In the sawtooth internal disruption process, four phases can be identified. The last three are characterized by the occurrence of an extra fluctuation which superimposes on the quasistationary turbulence. During the collapse phase, this phenomenon is very strong and localized inside the $q=1$ surface. The strong fluctuations cell has a radial extension a little less than $r_{1}$ and an azimuthal extension of one third of a turn. It is located at the cold area and coincides with the azimuthal location of the sooner and faster heat flux. The relative width of the $\omega$ spectrum is narrower than for QS turbulence, and suggests a quasi-coherent process. The typical frequency and phase velocity are about four times higher than that of the local quasi-stationary turbulence. The amplitude is two orders of magnitude larger than local QS and possibly could explain the high value of the heat diffusivity during the collapse.

These observations are in favour of the two fluids qualitative model proposed by Andreoletti. However in the model, the relaxation is the result of two interacting processes, one large scale : the kink, and one small scale : magnetodrift turbulence. Strictly speaking these two processes cannot be separated since the basic equations are nonlinear (mainly the momentum one), and as noted in the discussion experimental observations suggest that there may be no gap between the large scale and small scale parts of the motion. Moreover since the experimentally observed fluctuations show rather narrow spectra the small scale motions can hardly be considered as turbulence at least in the usual meaning of statistical fully developed turbulence. Then the kink motion $1 / 1$ with harmonics and the md fluctuations should evolve together selfconsistently as one global process in which the main role of small scale md fluctuations would be the breaking of flux conservation for the global convective motion.

According to the great difficulty of constructing such a theory, the only possibility appears to be a numerical simulation based on the two fluids nonlinear equations evolving in toroidal geometry. None of these aspects of the problem can be oversimplified if we want to reproduce and therefore understand the internal relaxation. The toroidal geometry, since in the mhd limit, calculations have shown that for $1 / 1$ kink the cylindrical terms identically cancel out. Full non linearity because coupling of the various scales is essential. Two fluids dynamics for the formation of some parallel electric field breaking magnetic flux conservation.

\section{Acknowledgements.}

The authors would like to thank TFR group's members for their active participation in the TFR experiments from which the present work was done. Thanks to C. Janicki, R. Decoste 
and $\mathrm{C}$. Simm for the reproduction of one figure from their article, and to J. How for his proof reading.

\section{References}

[1] Von Goeler S., Stodiek W., and Sauthoff N., Phys. Rev. Lett. 33 (1974) 1201.

[2] Shafranov V. D., Sov. Phys. Tech. Phys. 15 (1970) 175.

[3] Bussac M. N., Pellat R., Edery D. and Soule J. L., Phys. Rev. Lett. 35 (1975) 1638.

[4] Zakarov, Sov. J. Plasma Phys. 4 (1979) 503.

[5] Wesson J. A., Plasma Phys. Controlled Fusion 28 (1986) 243.

[6] Hastie R. J. and Hender T. C., Nucl. Fusion 28 (1988) 585.

[7] Waelbroeck F. L., Hazeltine R. D., Phys. Fluids 31 (1988) 1217.

[8] Furth H. P., Killeen J. and Rosenbluth M. N., Phys. Fluids 6 (1963) 459.

[9] Coppi B., Galvao R., Pellat R., Rosenbluth M. N. and Rutherford P., Sov. J. Plasma Phys. 2 (1976) 533.

[10] Bussac M. N., Edery D., Pellat R., Soule J. L., Plasma Phys. Contr. Nucl. Fusion, IAEA 1 (1977) 607.

[11] Coppi B., Englade R., Migliuolo S., Porcelli F. and Sugiyama L., Plasma Phys. Contr. Nucl. Fusion Res. (Kyoto 1986), IAEA (1987) Vol. 3, p. 397.

[12] Hastie R. J., Hender T. C., Carreras B. A., Charlton L. A. and Holmes J. A., Phys. Fluids 30 (1987) 1756.

[13] Parker E. N., J. Geophys. Res. 62 (1957) 509.

[14] Sweet P. A., Electromagnetic Phenomena in Cosmical Physics (Cambridge U.P., 1958), p. 123.

[15] Kadomtsev B. B., Sov. J. Plasma Phys. I (1975) 389.

[16] White R. B., Basic Plasma Physics, in Handbook of Plasma Physics, Vol. I, Chap. 3.5 (North Holland, 1983).

[17] Park W., Monticello D. A., White R. B., Phys. Fluids 27 (1984) 137.

[18] Rosenbluth M. N., Dagazian R. Y. and Rutherford P. H., Phys. Fluids 16 (1973) 1894.

[19] Aydemir A. Y., Phys. Rev. Lett. 59 (1987) 649.

[20] Holmes J. A., Carreras B. A., Charlton L. A., Lynch V. E., Hastie R. J. and Hender T. C., Phys. Fluids 31 (1988) 1202.

[21] Bussac M. N., Pellat R., Soule J. L. and Tagger M., Phys. Rev. Lett. A 105 (1984) 51 ; A 109 (1985) 331.

[22] Bussac M. N., Pellat R., Phys. Rev. Lett. 59 (1987) 2650.

[23] Danilov A. F., Dnestrovsky Y. N., Kostomarov D. P. and Popov A. M., Sov. J. Plasma Phys. 2 (1976) 93.

[24] Waddel B. V, Rosenbluth M. N., Monticello D. A. and White R. B., Nucl. Fusion 16 (1976) 528.

[25] Sykes A. and Wesson J. A., Phys. Rev. Lett. 37 (1976) 140.

[26] Denton R. E., Drake J. F., Kleva R. G. and Boyd D. A., Phys. Rev. Lett. 56 (1986) 2477.

[27] Kleva R. G., Drake J. F. and Denton R. E., Phys. Fluids 30 (1987) 2119.

[28] VLAD G. and Bondeson A., Nucl. Fusion 29 (1989) 1139.

[29] Samain A., Plasma Phys. 18 (1976) 551.

[30] Dubois M. A. and Samain A., Nucl. Fusion 20 (1980) 1101.

[31] Dubois M. A., Pecquet A. L., Reverdin C., Nucl. Fusion 23 (1983) 147.

[32] WeSsON J., Proceedings of Workshop on Theory of Fusion Plasmas, Varenna (1987). .

[33] Andreoletti J., in Turbulence and Nonlinear Dynamics in Mhd Flows (Elsevier Science Publishers, North-Holland, 1988), p. 177.

[34] Andreoletti J., Proc. 16th European Conference on Controlled Fusion and Plasma Physics (Venice 1989), Vol. 13B, Part I, p. 287.

[35] Mc Cormick K., Eberhagen A., Murmann H. et al., Proc. of 15th European Conf. on Controlled Fusion and Plasma Heating (Dubrovnik, 1988), Vol. 12B, Part I, p. 35.

[36] Weisen H., Borg G., Joyce B., Kinght A. J. and Lister J. B., Phys. Rev. Lett. 62 (1989) 434. 
[37] Soltwish H., Stodiek W., Manickam J. and Schluter J., Plasma Physics and Controlled Nuclear Fusion Research (Kyoto 1986), IAEA 1987, Vol. I, p. 263.

[38] West W. P., Thomas D. M., DE Grassie J. S. and Zheng S. B., Phys. Rev. Lett. 58 (1987) 2758.

[39] O'Rourke J. et al., Proc. 15th Eur. Conf. on Controlled Fusion and Plasma Heating (Dubrovnik 1988), Vol. 12B, p. 155.

[40] Gill R. D., Edwards A. W., Keegan B., Lazzaro E., O'Rourke J., Weller A. and Zasche D., Proc. 16th European Conference on Controlled Fusion and Plasma Physics (Venice 1989), Vol. 13B, Part. II. p. 469.

[41] Pegourie B., Dubois M. A. and Gill R. D., Proc. 16th European Conference on Controlled Fusion and Plasma Physics (Venice 1989), Vol. 13B, Part. II, p. 533.

[42] Campbell D. J. et al., Nucl. Fusion 26 (1986) 1085.

[43] Edwards A. W. et al., Phys. Rev. Lett. 57 (1986) 210.

[44] Campbell D. J. et al., Plasma Physics and Controlled Nuclear Fusion Research (Kyoto 1986), IAEA 1987, Vol. I, p. 433.

[45] Gill R. D. et al., Proc. 15th European Conference on Controlled Fusion and Plasma Heating (Dubrovnik 1988), Vol. 12B, 101 and JET Report-P (88), p. 15.

[46] Granetz R. S. and Smeulders P., Nucl. Fusion 28 (1988) 457.

[47] Janicki C., Decoste R. and Simm C., Phys. Rev. Lett. 62 (1989) 3038.

[48] Equipe TFR and Truc, A., C.R. Acad. Sci. Paris, Série II, 304 (1987) 255.

[49] TFR Group and TruC A., Gresillon D., Proc. 14th European Conference on Controlled Fusion and Plasma Physics (Madrid, 1987), Part I, 244.

[50] Andreoletti J., Gervais F., Olivain J., Quemeneur A., Truc A. and Gresillon D., Plasma Phys. Controlled Fusion 31 (1989) 643.

[51] Gervais F., Gresillon D., Hennequin P., Quemeneur A., Truc A., Andreoletti J., Laviron C., and Olivain J., Proc. 16th European Conference on Plasma Physics and Controlled Fusion (Venice, 1989), Vol. 13B, Part II, p. 497.

[52] TFR Group and Truc A., Plasma Phys. Controlled Fusion 26 (1984) 1045.

[53] TFR Group and TRUC A., Nucl. Fusion 26 (1986) 1303.

[54] Weisen H., Hollenstein C. H. and Behn R., Plasma Phys. and Controlled Fusion 30 (1988) 293.

[55] Barkley H., Gervais F., Olivain J., Quemeneur A. and Truc A., Proc. Int. Conf. on Plasma Physics (Kiev, 1987), Vol. 3, p. 24.

[56] Liewer P. C., Nucl. Fusion 25 (1985) 543.

[57] Hallock G. A., Mathew J., Jennings W. C. and Hickok R. L., Phys. Rev. Lett. 56 (1986) 1248. 